\title{
11. LATE MIOCENE TO HOLOCENE PLANKTONIC FORAMINIFERS FROM THE SUBANTARCTIC SOUTH ATLANTIC ${ }^{1}$
}

\author{
Claude Pujol ${ }^{2}$ and Robert Bourrouilh ${ }^{2}$
}

\begin{abstract}
Late Miocene to Holocene planktonic foraminifers from the subantarctic South Atlantic were investigated for their biostratigraphic and environmental significance.

The calcareous planktonic microfauna are of low diversity and are very rare or absent at sites located below the CCD. The sediments recovered from Sites 703 and 704 on the Meteor Rise at about $47^{\circ} \mathrm{S}$ are useful for biostratigraphic and environmental studies. In the whole sequence 16 species or varieties of planktonic foraminifers were recognized.

Two species occur in the uppermost Miocene. In the Pliocene the Globorotalia puncticulata population can be used to separate the early from the late Pliocene. The Pliocene/Quaternary boundary does not appear to be well distinguished in the foraminiferal assemblage.

A faunal change noted at $2.5 \mathrm{Ma}$ could correspond to the development of glaciation in the Northern Hemisphere and its Antarctic counterpart.

At about 5.2 Ma the first increase in polar fauna near the Meteor Rise occurs. Two other cooling periods are indicated in these sequences at about 4 and $3 \mathrm{Ma}$. Moreover, the hydrologic environment became more productive at about 2.1 Ma and close to the Brunhes/Matuyama boundary.
\end{abstract}

\section{INTRODUCTION}

The purpose of Ocean Drilling Program (ODP) Leg 114 was to study passageways in the subantarctic South Atlantic Ocean and oceanic current circulation. This paper will deal with late Miocene to Holocene foraminifers recovered from Holes 701A, 701C, 703A, 704A, and 704B (Table 1).

Site 701 is located to the east of the Islas Orcadas Rise, at $4644 \mathrm{~m}$ water depth, where the late Miocene to Holocene oceanic environment is a deep oceanic basin, swept by Antarctic Bottom Water (AABW). Sites 703 and 704 are located on the Meteor Rise, and their late Miocene to Holocene oceanic environment is one of an oceanic isolated rise. Site 703 is at $1817 \mathrm{~m}$ water depth in the core of North Atlantic Deep Water (NADW), while Site 704, at $2350 \mathrm{~m}$ water depth, is between NADW and Circumpolar Deep Water. All three sites are situated in the subantarctic zone to the north of present-day Polar Front Zone (PFZ) (Fig. 1).

The purpose of this study is to explore the planktonic foraminifers of the subantarctic South Atlantic Zone of the last $6 \mathrm{Ma}$ in order to determine the biostratigraphy of these sequences and to interpret the paleoclimatic evolution.

\section{MATERIALS AND METHODS}

Samples were prepared simply by drying and then soaking in water. This procedure was found to be sufficient to produce a complete breakdown of all the samples.

The samples were then washed through $150-$ and $63-\mu \mathrm{m}$ sieves and weighed. The percentage and composition of the coarse $(>150 \mu \mathrm{m})$ fraction are tabulated in the Appendix.

The analyses of planktonic foraminifers were made on the $>150-\mu \mathrm{m}$ fraction. A microsplitter was used to obtain a representative fraction of each sample for a frequency count

${ }^{1}$ Ciesielski, P. F., Kristoffersen, Y., et al., 1991. Proc. ODP, Sci. Results, 114: College Station, TX (Ocean Drilling Program).

${ }^{2}$ Département de Géologie et Océanographie, Université de Bordeaux 1, $3^{\circ}$ tranche-avenue des Facultés, 33405 Talence Cedex, France. based on no fewer than 300 specimens. The percentage distribution of the different species in each hole is summarized in Tables 2 through 4.

\section{SITE 701}

The three holes drilled east of Islas Orcadas Rise at Site 701 are located on Chron C22, that is, on oceanic crust of middle Eocene age. Hole 701A penetrated $74.8 \mathrm{~m}$ of Quaternary to lower Pliocene sediments with $93.13 \%$ recovery. Generally moderate drilling disturbance affected the first meters of the cored section (Ciesielski, Kristoffersen, et al., 1988).

Hole $701 \mathrm{C}$ penetrated $331 \mathrm{~m}$ of Quaternary to middle Eocene sediments with $66.1 \%$ recovery. Basalt was recovered at the bottom of the hole. Local drilling disturbance throughout the cores is generally moderate.

No major disconformity or erosional event appears in the upper part of the section, and the recent sedimentation seems to be quite continuous.

\section{Hole 701A}

The eight cores recovered with the advanced piston corer (APC) from Hole 701A represent a 71-m-long Pliocene-Quaternary sequence corresponding to the upper part of lithostratigraphic Subunit IA (Ciesielski, Kristoffersen, et al., 1988). The sequence consists of alternating intervals of ash-bearing diatom ooze and mud-bearing diatom ooze to diatom ooze. The base of the hole (Section 114-701A-8H-CC) is dated from diatoms (Fenner, this volume) at $3.10 \mathrm{Ma}$.

The 18 samples examined from Hole $701 \mathrm{~A}$ in this study yielded a chiefly siliceous component (Fig. 2 and Appendix).

Only Samples 114-701A-3H-6, 90-92 cm, 114-701A-5H-4, $34-36 \mathrm{~cm}$, and $114-701 \mathrm{~A}-5 \mathrm{H}-5,34-36 \mathrm{~cm}$, contain a monospecific assemblage of the planktonic foraminifer species Neogloboquadrina pachyderma (left-coiling form). The samples with this polar species (Bé, 1977) are dated by the extrapolation of siliceous microfossil datums (Ciesielski, Kristoffersen, et al., 1988) to $1.15,1.64$, and $1.70 \mathrm{Ma}$, respectively.

It is well known that in the deep-sea environment the corrosiveness of the deep waters determine the degree of 
Table 1. Location and water depth of investigated Leg 114 sites.

\begin{tabular}{lccc}
\hline Hole & Latitude & Longitude & Water depth (m) \\
\hline $701 \mathrm{~A}$ & $51^{\circ} 59.076^{\prime} \mathrm{S}$ & $23^{\circ} 12.736^{\prime} \mathrm{W}$ & 4647.2 \\
$701 \mathrm{C}$ & $51^{\circ} 59.085^{\prime} \mathrm{S}$ & $23^{\circ} 12.700^{\prime} \mathrm{W}$ & 4647.2 \\
$703 \mathrm{~A}$ & $47^{\circ} 03.042^{\prime} \mathrm{S}$ & $07^{\circ} 53.679^{\prime} \mathrm{E}$ & 1806.6 \\
$704 \mathrm{~A}$ & $46^{\circ} 52.757^{\prime} \mathrm{S}$ & $07^{\circ} 53.679^{\prime} \mathrm{E}$ & 1806.6 \\
$704 \mathrm{~B}$ & $46^{\circ} 52.758^{\prime} \mathrm{S}$ & $07^{\circ} 25.231^{\prime} \mathrm{E}$ & 2530.8 \\
\hline
\end{tabular}

dissolution of calcareous deposits and the distribution of biologic remains on the seafloor. This may explain the absence of foraminifers in most of the samples studied. The cold-water assemblage recognized between 1.70 to $1.15 \mathrm{Ma}$ in the lower Quaternary may be interpreted as indicative of a reduced corrosiveness of AABW.

\section{Hole 701C}

Eighteen samples were examined from APC-recovered Cores 114-701C-17H through 114-701C-7H. The sedimentary sequence is latest Miocene and Pliocene in age, from about 6.6 to $1.86 \mathrm{Ma}$ (Ciesielski, Kristoffersen, et al., 1988).

This sequence is attributed to lithostratigraphic Subunits IA and IB. The lithology of Subunit IA is similar to that described previously for Hole 701A. Subunit IB (Core 114$701 \mathrm{C}-17 \mathrm{H})$ consists of finely laminated diatom ooze, dominated by the diatom species Bruniopsis mirabilis (Ciesielski, Kristoffersen, et al., 1988). This whole sequence is completely barren of planktonic foraminifers (Fig. 3 and Appendix). As for Hole 701A, the lack of carbonate microfossils within the upper Miocene and the whole Pliocene section of sediments at this site indicates the corrosiveness of the bottom water below the carbonate compensation depth (CCD).

\section{SITE 703}

\section{Hole 703A}

A single hole was drilled at Site 703, located on the slope of a basement high of the Meteor Rise, in a water depth of 1796.1 m.

Hole 703A penetrated $377.4 \mathrm{~m}$, with an average recovery of $84.6 \%$ in the first $190 \mathrm{~m}$ and very poor recovery (17.7\%) below that depth. The Quaternary to middle Eocene sediments consist of calcareous ooze and chalk. The Quaternary and upper Pliocene section contains volcanic ash-bearing, nannofossil foraminifer ooze and rests unconformably on lower Miocene sediments. Thus, an important pre-late Pliocene event of erosion or nondeposition characterizes this site and appears to affect the recent depositional history of this area of the Meteor Rise.

Unfortunately, drilling disturbance is very strong in the Quaternary to upper Pliocene sediments, which are soupy to highly disturbed. The three samples analyzed from highrecovery $(100 \%)$ Core $114-703 \mathrm{~A}-1 \mathrm{H}$ correspond to a late Quaternary interval dated from 0.8 to $0.1 \mathrm{Ma}$ by the extrapolation of diatom datums (Fenner, this volume). The sediment of lithostratigraphic Subunit IA consists of a siliceous-foraminifer-bearing nannofossil ooze (Ciesielski, Kristoffersen, et al., 1988). Because of the relatively shallow water depth (1807 $\mathrm{m})$ this site location is above the CCD, and the coarse fraction of the samples investigated consists of more than $90 \%$ planktonic foraminifers (Appendix).

The assemblage from Hole 703A comprises Globigerina bulloides, Globigerina quinqueloba, Globorotalia inflata, and Globorotalia truncatulinoides and is associated with the dominant cold-water, left-coiling form Neogloboquadrina pachy- derma (Table 2), which indicates an assemblage typical of a subantarctic water mass.

G. puncticulata, which was observed in Samples 114$703 \mathrm{~A}-1 \mathrm{H}-2,140-142 \mathrm{~cm}$, and $114-703 \mathrm{~A}-1 \mathrm{H}-3,140-142 \mathrm{~cm}$, is dated by the extrapolation of siliceous microfossil datums (Ciesielski, Kristoffersen, et al., 1988) to 0.6 and $0.8 \mathrm{Ma}$, respectively.

\section{SITE 704}

Two holes were drilled at Site 704 on the western flank of the Meteor Rise at a water depth of $2532.3 \mathrm{~m}$. Hole 704A penetrated $282.7 \mathrm{~m}$ of upper Miocene to upper Quaternary sediments with an average recovery of $79.4 \%$. Drilling disturbance is locally strong in Cores $114-704 \mathrm{~A}-1 \mathrm{H}, 114-704 \mathrm{~A}-4 \mathrm{H}$, and $114-704 \mathrm{~A}-5 \mathrm{H}$. Hole $704 \mathrm{~B}$ penetrated $671.7 \mathrm{~m}$ of lower Oligocene to upper Quaternary sediments, with an average recovery of $74.8 \%$.

\section{Hole 704A}

Planktonic foraminifers are abundant and well preserved within the 230 -m-long sequence investigated from Hole $704 \mathrm{~A}$ (Fig. 4 and Appendix). The very few signs of dissolution indicate a relatively shallow depth of deposition.

The lithology of Hole 704A (Ciesielski, Kristoffersen, et al., 1988) comprises calcareous-bearing diatom ooze of lithostratigraphic Subunit IA (Cores 114-704A-1H to 114-704A$14 \mathrm{H}$ ), siliceous calcareous ooze of Subunit IB (Cores 114$704 \mathrm{~A}-15 \mathrm{H}$ to $114-704 \mathrm{~A}-22 \mathrm{X}$ ), and diatom-bearing calcareous ooze of Subunit IC (Cores 114-704A-21X to 114-704A-25X).

Sixteen species or varieties of planktonic foraminifers were recognized (Table 3 ). The abundance of planktonic foraminifers (expressed as the number of specimens per $10 \mathrm{~g}$ of dry sediment in the fraction $>150 \mu \mathrm{m}$ ) shows two distinct phases (Fig. 5). The first phase, from the base of the hole to about 169 $\mathrm{m}$ below seafloor (mbsf), has few foraminifers (always less than $10^{6}$ specimens $/ 10 \mathrm{~g}$ of dry sediment) and is without major fluctuations. The second phase, between 169 and $1.15 \mathrm{mbsf}$ (from Sample 114-704A-19X-1, 40-42 cm, to the top of our sampling interval), varies widely and has abundance peaks of more than $4 \times 10^{5}$ specimens $/ 10 \mathrm{~g}$ of sediment. The transition between these two phases is dated to about $2.5 \mathrm{Ma}$.

Fluctuations of the carbonate content confirm the division of the lithostratigraphic units (Ciesielski, Kristoffersen, et al., 1988), but they do not correlate with the abundance curve of the calcareous planktonic foraminifers.

In Figure 6, the percentage of the different species in the coarse fraction is plotted against an extrapolated chronological scale derived from biostratigraphic and paleomagnetic datums (Ciesielski, Kristoffersen, et al., 1988). In the upper Miocene sequence (Core 114-704A-25X) some specimens of Globorotalia cf. sphericonomiozea were observed. This form disappears at about $5.35 \mathrm{Ma}$, dated by the extrapolation of microfossil and paleomagnetic datums (Ciesielski, Kristoffersen, et al., 1988). The overlying lower Pliocene sequence contains Globorotalia cf. juanai, but some specimens were also recognized at the same levels as $G$. cf. sphericonomiozea. The last-appearance datum (LAD) of $G$. aff. juanai (Sample 114-704A-23X-5, 10-12 cm), at the base of the Sidufjall Subchron (Ciesielski, Kristoffersen, et al., 1988), is dated to about 4.5 Ma. In sediments younger than $2.5 \mathrm{Ma}$, the planktonic foraminiferal fauna consists of two alternating groups, Neogloboquadrina pachyderma (left-coiling form) and Globigerina bulloides, Globorotalia puncticulata, and, to a lesser degree, Globigerinita glutinata (Fig. 6).

The polar, left-coiling form $N$. pachyderma is the dominant species in the whole sequence. It represents $50 \%$ to $90 \%$ of the whole association in the upper section of the hole ( 0.4 to 2.5 

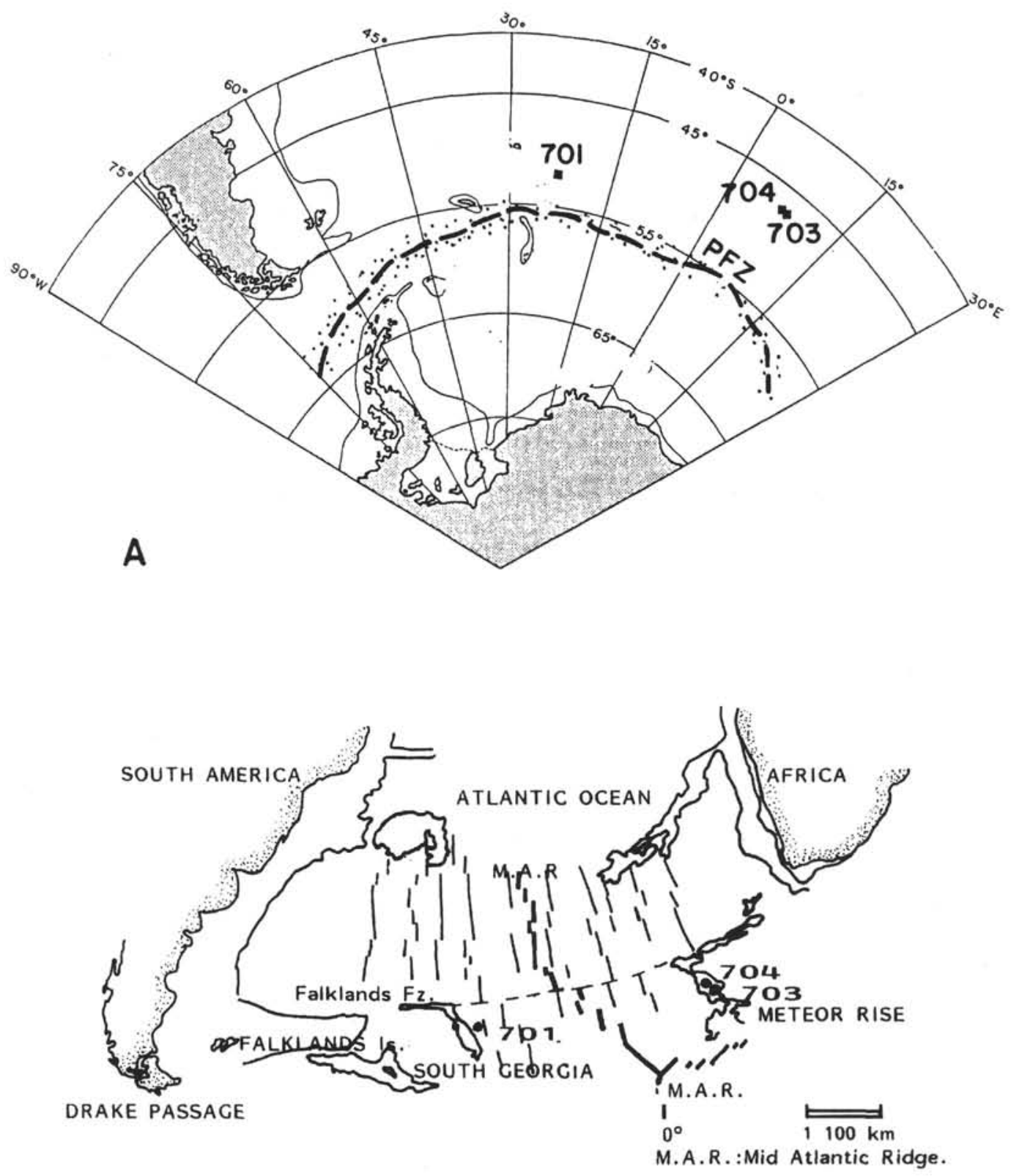

B

Figure 1. Location of the investigated Leg 114 sites. A. Site position with respect to the Polar Front Zone (PFZ). B. Map of the subantarctic South Atlantic.

Table 2. Distribution and relative abundance of planktonic foraminifers, Hole 703A.

\begin{tabular}{|c|c|c|c|c|c|c|c|c|c|c|}
\hline $\begin{array}{l}\text { Core, section, } \\
\text { interval }(\mathrm{cm})\end{array}$ & $\begin{array}{l}\text { Depth } \\
\text { (mbsf) }\end{array}$ & $\begin{array}{l}\text { Age } \\
\text { (Ma) }\end{array}$ & $\begin{array}{c}\text { Specimens } \\
\text { per } 10 \mathrm{~g}\end{array}$ & $\begin{array}{c}N . \\
\text { pachyderma, } \\
\text { left-coiling }\end{array}$ & $\begin{array}{c}N . \\
\text { pachyderma, } \\
\text { right-coiling }\end{array}$ & $\begin{array}{c}G . \\
\text { bulliodes }\end{array}$ & $\begin{array}{c}G . \\
\text { inflata }\end{array}$ & $\begin{array}{c}G . \\
\text { quinqueloba }\end{array}$ & $\begin{array}{c}G . \\
\text { truncatulinoides }\end{array}$ & $\begin{array}{c}G . \\
\text { puncticulata }\end{array}$ \\
\hline \multicolumn{11}{|l|}{ 114-703A- } \\
\hline $1 \mathrm{H}-1,50-52$ & 0.5 & 0.1 & 956,300 & 91.1 & 1.00 & 3.0 & 0.2 & 4.4 & \multirow{3}{*}{7.0} & \\
\hline $1 \mathrm{H}-2,140-142$ & 2.9 & 0.6 & 666,700 & 89.5 & 0.70 & 3.0 & 0.7 & 6.0 & & 23.0 \\
\hline $1 \mathrm{H}-3,140-142$ & 4.4 & 0.8 & 907,300 & 73.5 & 1.0 & 10.7 & 1.0 & 1.0 & & 10.3 \\
\hline
\end{tabular}


Table 3. Distribution and relative abundance of planktonic foraminifers, Hole 704A

\begin{tabular}{|c|c|c|c|c|c|c|c|c|c|c|c|c|c|c|c|c|c|c|c|}
\hline $\begin{array}{l}\text { Core, section, } \\
\text { interval (cm) }\end{array}$ & $\begin{array}{l}\text { Depth } \\
\text { (mbsf) }\end{array}$ & $\begin{array}{l}\text { Age } \\
\text { (Ma) }\end{array}$ & $\begin{array}{c}\text { Specimens } \\
\text { per } 10 \mathrm{~g}\end{array}$ & $\begin{array}{c}N . \\
\text { pachy- } \\
\text { derma, } \\
\text { left- } \\
\text { coiling }\end{array}$ & $\begin{array}{c}N . \\
\text { pachy- } \\
\text { derma, } \\
\text { sp. } 1 \text {, } \\
\text { left- } \\
\text { coiling }\end{array}$ & $\begin{array}{l}N . \\
\text { pachy- } \\
\text { derma } \\
\text { right- } \\
\text { coiling }\end{array}$ & $\begin{array}{c}G . \\
\text { bulliodes }\end{array}$ & $\begin{array}{c}G . \text { aff. } \\
\text { bulloides }\end{array}$ & $\begin{array}{c}G . \\
\text { glutinata }\end{array}$ & $\begin{array}{c}G . \\
\text { quinque- } \\
\text { loba }\end{array}$ & $\underset{\text { scitula }}{G .}$ & $\begin{array}{c}\text { G. } \\
\text { punctic- } \\
\text { ulata }\end{array}$ & $\begin{array}{l}\text { G. aff. } \\
\text { punctic- } \\
\text { ulata }\end{array}$ & $\begin{array}{c}G . \\
\text { aff. } \\
\text { juanai }\end{array}$ & $\begin{array}{l}G . \text { aff. } \\
\text { spherico- } \\
\text { nomiozea }\end{array}$ & $\begin{array}{c}G . \\
\text { crassa- } \\
\text { formis }\end{array}$ & $\begin{array}{c}G . \\
\text { inflata }\end{array}$ & $\begin{array}{c}\text { G. } \\
\text { truncatu- } \\
\text { linoides }\end{array}$ & $\begin{array}{c}G . \\
\text { uvula }\end{array}$ \\
\hline \multicolumn{20}{|l|}{ 119-704A- } \\
\hline $1 \mathrm{H}-1,115-117$ & 1.15 & 0.40 & 395,000 & 92.0 & & 0.3 & 6.0 & & 0.3 & 0.6 & & & & & & & 1.0 & 1.7 & \\
\hline $1 \mathrm{H}-2,115-117$ & 2.65 & 0.41 & 137,000 & 26.0 & & 1.7 & 58.0 & & 2.3 & & + & & & & & 8.6 & 10.9 & & \\
\hline $1 \mathrm{H}-4,115-117$ & 5.65 & 0.44 & 21,200 & 39.6 & & 0.3 & 31.3 & & 8.3 & 0.6 & & & & & & & 0.5 & & \\
\hline $2 \mathrm{H}-2,114-116$ & 9.80 & 0.48 & 1,030 & 94.5 & & & 2.5 & & 2.5 & & & 1.0 & & & & & & & \\
\hline $2 \mathrm{H}-4,114-115$ & 12.80 & 0.52 & 628,000 & 99.6 & & & 0.2 & & & 0.2 & & & & & & & & & \\
\hline $2 \mathrm{H}-6,114-116$ & 15.80 & 0.55 & 85,400 & 99.4 & & & 0.6 & & & & & + & & & & & & & \\
\hline $3 \mathrm{H}-2,135-137$ & 19.55 & 0.58 & 29,500 & 74.6 & & & 4.7 & & 0.4 & & & 20.0 & & & & & & & \\
\hline $3 \mathrm{H}-4,135-137$ & 22.55 & 0.61 & 188,000 & 76.7 & & & 21.0 & & & 0.8 & & 1.3 & & & & & & & \\
\hline $3 \mathrm{H}-6,135-137$ & 25.55 & 0.64 & 287,000 & 87.8 & & & 5.8 & & & 0.9 & & 5.3 & & & & & & & \\
\hline $4 \mathrm{H}-3,10-12$ & 29.30 & 0.69 & 920,000 & 94.0 & & & & & & 5.7 & 0.3 & & & & & & & & \\
\hline $4 \mathrm{H}-6,10-12$ & 33.70 & 0.73 & 510,000 & 87.5 & & & 1.7 & & 0.5 & 2.6 & 0.1 & 7.2 & & & & & & & \\
\hline $5 \mathrm{H}-6,0-2$ & 43.20 & 0.86 & 250,000 & 88.4 & & & 1.6 & & & & & 9.8 & & & & & & & \\
\hline $5 \mathrm{H}-7,0-2$ & 44.70 & 0.88 & 63,000 & 99.6 & & & 0.0 & & & 0.2 & 0.2 & & & & & & & & \\
\hline $6 \mathrm{H}-2,0-2$ & 46.70 & 0.91 & 94,600 & 77.3 & & & 3.2 & & 0.5 & & 0.8 & 18.0 & & & & & & & \\
\hline $6 \mathrm{H}-4,0-2$ & 49.70 & 0.95 & 65,000 & 97.8 & & & & & & & & 2.0 & & & & & & & \\
\hline $8 \mathrm{H}-2,21-23$ & 65.91 & 1.20 & 8,800 & 72.0 & 27.50 & & & & & & & 0.4 & & & & & & & \\
\hline $8 \mathrm{H}-4,21-23$ & 68.91 & 1.24 & 7,200 & 89.3 & 5.30 & & 0.8 & & & 0.8 & & 3.5 & & & & & & & \\
\hline $8 \mathrm{H}-6,18-20$ & 71.88 & 1.28 & 44,500 & 73.0 & 15.00 & & 0.9 & & 0.2 & 0.2 & & 9.7 & & & & & & & \\
\hline $9 \mathrm{H}-1,76-78$ & 74.46 & 1.31 & 69,300 & 61.3 & 32.10 & & 0.5 & 0.5 & & & & 5.3 & & & & & & & \\
\hline $9 \mathrm{H}-4,76-78$ & 78.96 & 1.37 & 457,000 & 55.6 & 22.00 & & 6.0 & 1 & 0.1 & 1.0 & & 14.0 & & & & & & & \\
\hline $9 \mathrm{H}-7,30-32$ & 83.00 & 1.43 & 26,400 & 61.2 & 31.80 & & 2.8 & 2.4 & & 0.4 & & 1.2 & & & & & & & 0.3 \\
\hline $10 \mathrm{H}-1,90-92$ & 84.10 & 1.45 & 6,400 & 96.8 & & & 2.5 & & & & & 0.3 & & & & & & & \\
\hline $10 \mathrm{H}-3,90-92$ & 87.10 & 1.50 & 2,100 & 80.7 & & & & & & 0.5 & & 9.6 & & & & & & & \\
\hline $10 \mathrm{H}-5,90-92$ & 90.10 & 1.55 & 30 & & & & & & & & & & & & & & & & \\
\hline $11 \mathrm{H}-1,104-406$ & 93.74 & 1.66 & 33,300 & 92.2 & & & 1.5 & & 0.2 & & & 6.0 & & & & & & & \\
\hline $11 \mathrm{H}-4,104-406$ & 97.24 & 1.69 & 332,000 & 76.4 & & & 6.8 & 1 & 0.4 & 0.8 & 0.2 & 14.0 & & & & & & & \\
\hline $11 \mathrm{H}-7,47-49$ & 102.17 & 1.73 & 515,000 & 91.3 & & & 4.7 & & 0.5 & & 0.4 & 3.0 & & & & & & & \\
\hline $12 \mathrm{H}-1,130-132$ & 103.50 & 1.74 & 107,000 & 72.5 & & & 2.3 & 2.3 & & & 0.4 & 22.2 & & & & & & & \\
\hline $12 \mathrm{H}-3,130-132$ & 106.50 & 1.76 & 143,000 & 75.7 & & & 6.0 & & 1.1 & & 0.5 & 16.2 & & & & & & & \\
\hline $12 \mathrm{H}-6,130-132$ & 109.50 & 1.80 & 285,000 & 43.5 & & & 18.5 & 1.3 & & & 0.2 & 36.0 & & & & & & & \\
\hline $13 \mathrm{H}-1,90-92$ & 112.60 & 1.84 & 96,000 & 93.0 & & & & 6 & & & & 0.8 & & & & & & & \\
\hline $13 \mathrm{H}-3,90-92$ & 115.60 & 1.87 & 74,000 & 90.8 & & & 0.5 & & 0.5 & & & 8.3 & & & & & & & \\
\hline $13 \mathrm{H}-5,90-92$ & 118.60 & 1.90 & 271,000 & 92.8 & & & 3.0 & & & & 0.2 & 3.8 & & & & & & & \\
\hline $14 \mathrm{H}-1,90-92$ & 112.10 & 1.99 & 623,000 & 78.6 & & & 5.6 & & & & 0.7 & 14.8 & & & & & & & \\
\hline $14 \mathrm{H}-3,90-92$ & 125.10 & 2.02 & 82,700 & 74.6 & & & 5.3 & & 0.8 & 1.1 & & 18.0 & & & & & & & \\
\hline $14 \mathrm{H}-5,90-92$ & 128.10 & 2.05 & 111,000 & 74.0 & & & 8.6 & & 1 & & 1.6 & 14.5 & & & & & & & \\
\hline
\end{tabular}


Table 3 (continued).

\begin{tabular}{|c|c|c|c|c|c|c|c|c|c|c|c|c|c|c|c|c|c|c|c|}
\hline $\begin{array}{l}\text { Core, section, } \\
\text { interval }(\mathrm{cm})\end{array}$ & $\begin{array}{l}\text { Depth } \\
\text { (mbsf) }\end{array}$ & $\begin{array}{l}\text { Age } \\
\text { (Ma) }\end{array}$ & $\begin{array}{c}\text { Specimens } \\
\text { per } 10 \mathrm{~g}\end{array}$ & $\begin{array}{l}N . \\
\text { pachy- } \\
\text { derma, } \\
\text { left- } \\
\text { coiling }\end{array}$ & $\begin{array}{c}N . \\
\text { pachy- } \\
\text { derma, } \\
\text { sp. } 1 \text {, } \\
\text { left- } \\
\text { coiling }\end{array}$ & $\begin{array}{l}N . \\
\text { pachy- } \\
\text { derma } \\
\text { right- } \\
\text { coiling }\end{array}$ & $\begin{array}{c}G . \\
\text { bulliodes }\end{array}$ & $\begin{array}{c}G . \text { aff. } \\
\text { bulloides }\end{array}$ & $\begin{array}{c}G . \\
\text { glutinata }\end{array}$ & $\begin{array}{c}G . \\
\text { quinque- } \\
\text { loba }\end{array}$ & $\begin{array}{c}G . \\
\text { scitula }\end{array}$ & $\begin{array}{c}G . \\
\text { punctic- } \\
\text { ulata }\end{array}$ & $\begin{array}{l}\text { G. aff. } \\
\text { punctic- } \\
\text { ulata }\end{array}$ & $\begin{array}{c}G . \\
\text { aff. } \\
\text { juanai }\end{array}$ & $\begin{array}{c}\text { G. aff. } \\
\text { spherico- } \\
\text { nomiozea }\end{array}$ & $\begin{array}{c}G . \\
\text { crassa- } \\
\text { formis }\end{array}$ & $\begin{array}{c}G . \\
\text { inflata }\end{array}$ & $\begin{array}{c}\text { G. } \\
\text { truncatu- } \\
\text { linoides }\end{array}$ & $\begin{array}{c}G . \\
\text { uvula }\end{array}$ \\
\hline $15 \mathrm{H}-2,90-92$ & 132.10 & 2.10 & 49,200 & 84.2 & & & 10.0 & & & 1.5 & & 4.1 & & & & & & & \\
\hline $15 \mathrm{H}-4,90-92$ & 135.10 & 2.13 & 82,900 & 54.7 & & & 30.0 & & 0.2 & 0.3 & 0.2 & 14.3 & & & & & & & \\
\hline $15 \mathrm{H}-6,90-92$ & 138.10 & 2.16 & 148,000 & 28.1 & & & 32.6 & & 2 & 6.0 & & 31.0 & & & & & & & \\
\hline $16 \mathrm{H}-2,16-18$ & 140.16 & 2.20 & 1,300 & 28.5 & & & 2.8 & & & & & 68.0 & & & & & & & \\
\hline $16 \mathrm{H}-4,16-18$ & 143.16 & 2.23 & 117,500 & 80.3 & & & 15.7 & & & & 0.2 & 3.6 & & & & & & & \\
\hline $16 \mathrm{H}-6,16-18$ & 146.16 & 2.26 & 12,300 & 41.5 & & & 28.3 & 4.1 & 2 & 1.0 & 0.3 & 20.4 & & & & & & & \\
\hline $17 \mathrm{H}-1,116-118$ & 150.86 & 2.30 & 50,700 & 70.8 & & & 7.0 & & & & 0.2 & 19.9 & & & & & & & \\
\hline $17 \mathrm{H}-3,116-118$ & 153.86 & 2.34 & 330,000 & 92.3 & & & 5.4 & & 0.3 & & 0.2 & 1.6 & & & & & & & \\
\hline $17 \mathrm{H}-5,116-118$ & 156.86 & 2.37 & 131,200 & 82.9 & & & 10.0 & & 0.2 & & & 6.5 & & & & & & & \\
\hline $18 X-2,36-38$ & 161.06 & 2.40 & 55,100 & 88.2 & & & 2.6 & & & & 0.2 & 8.8 & & & & & & & \\
\hline $18 \mathrm{X}-4,36-38$ & 164.06 & 2.43 & 60,400 & 97.1 & & & 1.1 & & & & & 1.7 & & & & & & & \\
\hline $18 \mathrm{X}-6,36-38$ & 167.06 & 2.47 & 7,800 & 53.7 & & & 7.4 & & 0.4 & & & 38.4 & & & & & & & \\
\hline $19 \mathrm{X}-1,40-42$ & 169.10 & 2.59 & 12,800 & 41.0 & & & 3.8 & & & & & 55.0 & & & & & & & \\
\hline $19 \mathrm{X}-3,40-42$ & 172.10 & 2.75 & 12,200 & 03.6 & & & 4.5 & & 1.8 & & & 90.0 & & & & & & & \\
\hline $19 X-5,40-42$ & 175.10 & 2.92 & 33,600 & 56.1 & & & 34.4 & 2.1 & 2.1 & 0.1 & & 5.0 & & & & & & & \\
\hline $20 \mathrm{X}-1,130-132$ & 179.50 & 3.08 & 58,800 & 42.5 & & & 25.5 & 3.7 & 8.1 & & 0.1 & 20.2 & & & & & & & \\
\hline $20 \mathrm{X}-3,130-132$ & 182.50 & 3.18 & 300 & 02.3 & & & 1.7 & & 2.3 & & & 93.0 & & & & & & & \\
\hline $20 \mathrm{X}-5,130-132$ & 185.50 & 3.30 & 59,400 & 06.7 & & & 5.0 & 2.1 & 5.4 & & 0.4 & 80.0 & & & & & & & \\
\hline $21 X-1,70-72$ & 187.40 & 3.40 & 131,400 & 00.6 & & & 37.4 & 0.3 & 1.2 & & 10.8 & 1.8 & 4.8 & & & & & & \\
\hline $21 X-3,70-72$ & 190.40 & 3.54 & 54,000 & 02.5 & & & 45.6 & & 1.7 & 0.4 & 8.6 & 6.0 & 35 & & & & & & \\
\hline $22 \mathrm{X}-1,104-106$ & 198.24 & 3.88 & 15,000 & 22.7 & & & 3.6 & 0.7 & 5.8 & & 0.7 & & 53.1 & & & & & & \\
\hline $22 \mathrm{X}-3,104-106$ & 201.24 & 3.99 & 170 & 71.0 & & & 3.0 & 24 & 2.0 & & & & & & & & & & \\
\hline $22 \mathrm{X}-5,104-106$ & 204.24 & 4.10 & 170 & 28.5 & & & 64.0 & 20.5 & 3.0 & & & & 9 & & & & & & \\
\hline $23 \mathrm{X}-1,10-12$ & 206.80 & 4.15 & 8,400 & 25.0 & & & 29.0 & 3.8 & 2.5 & & & & 37.5 & & & & & & \\
\hline $23 \mathrm{X}-3,10-12$ & 209.80 & 4.24 & 34,600 & 12.3 & & & 73.8 & 6.1 & & & 0.6 & & 7 & & & & & & \\
\hline $23 \mathrm{X}-5,10-12$ & 212.80 & 4.52 & 11,000 & 19.5 & & + & 56.2 & & 1.4 & & 0.2 & & 20 & 2 & & & & & \\
\hline $24 \mathrm{X}-1,0-2$ & 216.20 & 4.80 & 44.3 & & & & 20.0 & & 4.4 & & & & 12.2 & 18.7 & & & & & \\
\hline $24 \mathrm{X}-3,0-2$ & 219.20 & 5.01 & 600 & 19.9 & & & 16.4 & 3.5 & 3.5 & & 1.0 & & 45.8 & 10.5 & & & & & \\
\hline $24 X-5,0-2$ & 222.20 & 5.23 & 6,700 & 73.2 & & & 5.4 & 19.6 & 1.5 & & & & & + & & & & & \\
\hline $25 \mathrm{X}-1,0-2$ & 225.70 & 5.35 & 800 & 39.0 & & & 9.0 & 25 & 5.8 & & & & & 9.1 & 6.4 & & & & \\
\hline $25 \mathrm{X}-3,0-2$ & 228.70 & 5.56 & 5 & + & & & & + & & & & & & & & & & & \\
\hline $25 \mathrm{X}-5,0-2$ & 231.70 & 5.77 & 700 & 00.4 & & & 87.0 & & 9.4 & 0.4 & 1.2 & & & + & 1.2 & & & & \\
\hline
\end{tabular}


Table 4. Distribution and relative abundance of planktonic foraminifers, Hole 704B.

\begin{tabular}{|c|c|c|c|c|c|c|c|c|c|}
\hline $\begin{array}{l}\text { Core, section, } \\
\text { interval }(\mathrm{cm})\end{array}$ & $\begin{array}{l}\text { Depth } \\
\text { (mbsf) }\end{array}$ & $\begin{array}{l}\text { Age } \\
(\mathrm{Ma})\end{array}$ & $\begin{array}{l}\text { Specimens } \\
\text { per } 10 \mathrm{~g}\end{array}$ & $\begin{array}{l}\text { N. pachyderma, } \\
\text { left-coiling }\end{array}$ & $\begin{array}{l}\text { N. pachyderma, } \\
\text { right-coiling }\end{array}$ & G. bulliodes & G. quinqueloba & G. puncticulata & G. uvula \\
\hline \multicolumn{10}{|l|}{ 114-704B- } \\
\hline $4 \mathrm{H}-2,40-42$ & 26.6 & 0.6 & 391,700 & 83.0 & 0.5 & 12.6 & 1.4 & 2.0 & 0.5 \\
\hline $4 \mathrm{H}-4,40-42$ & 29.6 & 0.69 & 61,000 & 77.0 & & 21.0 & 1.4 & 0.5 & 0.5 \\
\hline $4 \mathrm{H}-6,40-42$ & 32.6 & 0.7 & 96,700 & 97.0 & & 1.3 & & 1.7 & \\
\hline $5 \mathrm{H}-1,115-117$ & 36.35 & 0.8 & 400,000 & 78.0 & & 13.0 & 2.6 & 6.0 & 0.2 \\
\hline
\end{tabular}

Components (\%)

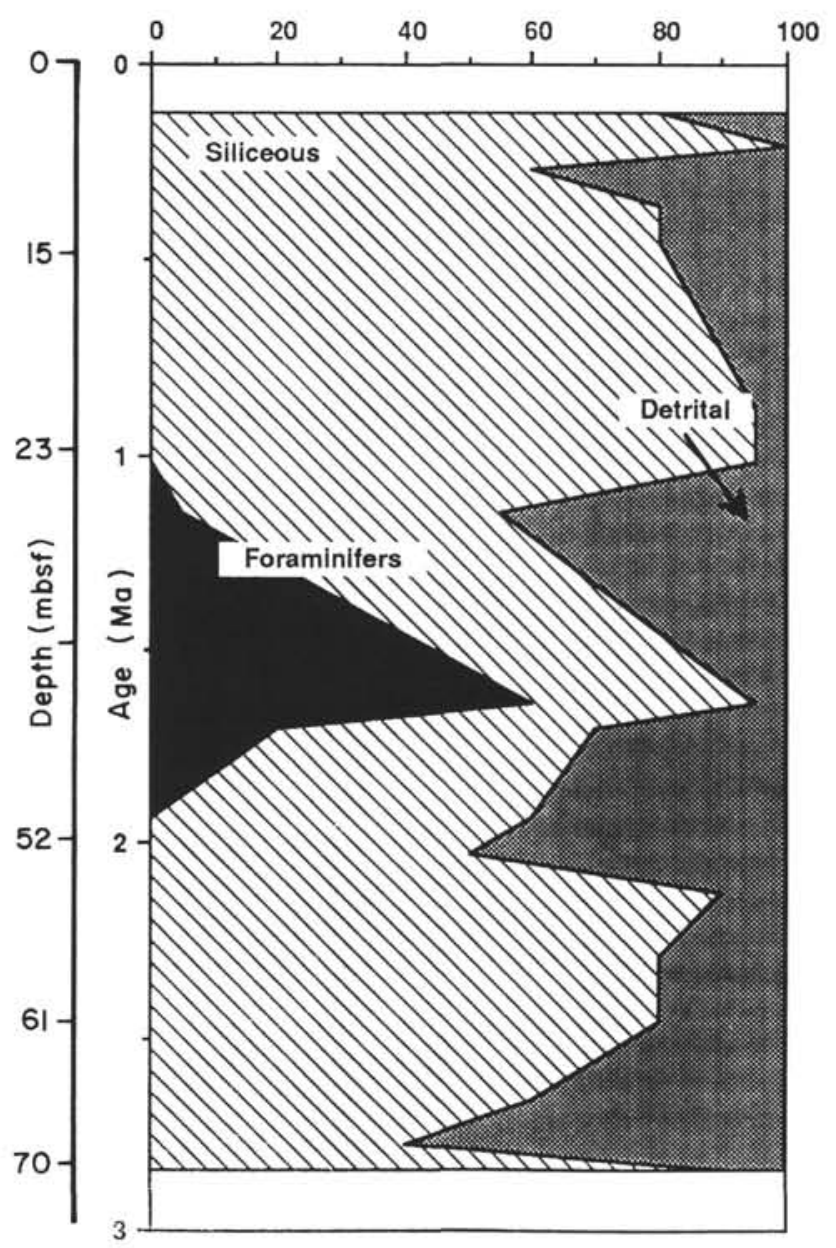

Figure 2. Principal components of the coarse $(>150 \mu \mathrm{m})$ fraction, Hole 701A (0-70 mbsf).

Ma). Within the lower part of the hole abundance maxima are always lower than $40 \%-60 \%$, with broader variations than in the upper part. A few specimens of the right-coiling form of $N$. pachyderma were observed only in Core 114-704A-1H (Table 3). Another interesting species distribution is that of $G$. bulloides. This species has a good representation $(10 \%$ to more than $50 \%$ ) from Cores $114-704 \mathrm{~A}-25 \mathrm{X}$ to $114-704 \mathrm{~A}-19 \mathrm{X}$, with a frequency decrease at about $2.5 \mathrm{Ma}$ (169 mbsf). The distribution of $G$. puncticulata s.l. appears similar to that of $G$. bulloides. This species appears at about $5 \mathrm{Ma}$ (Sample 114704A-24X-3, 0-2 cm) and disappears at about 0.5 Ma (Sample
Components (\%)

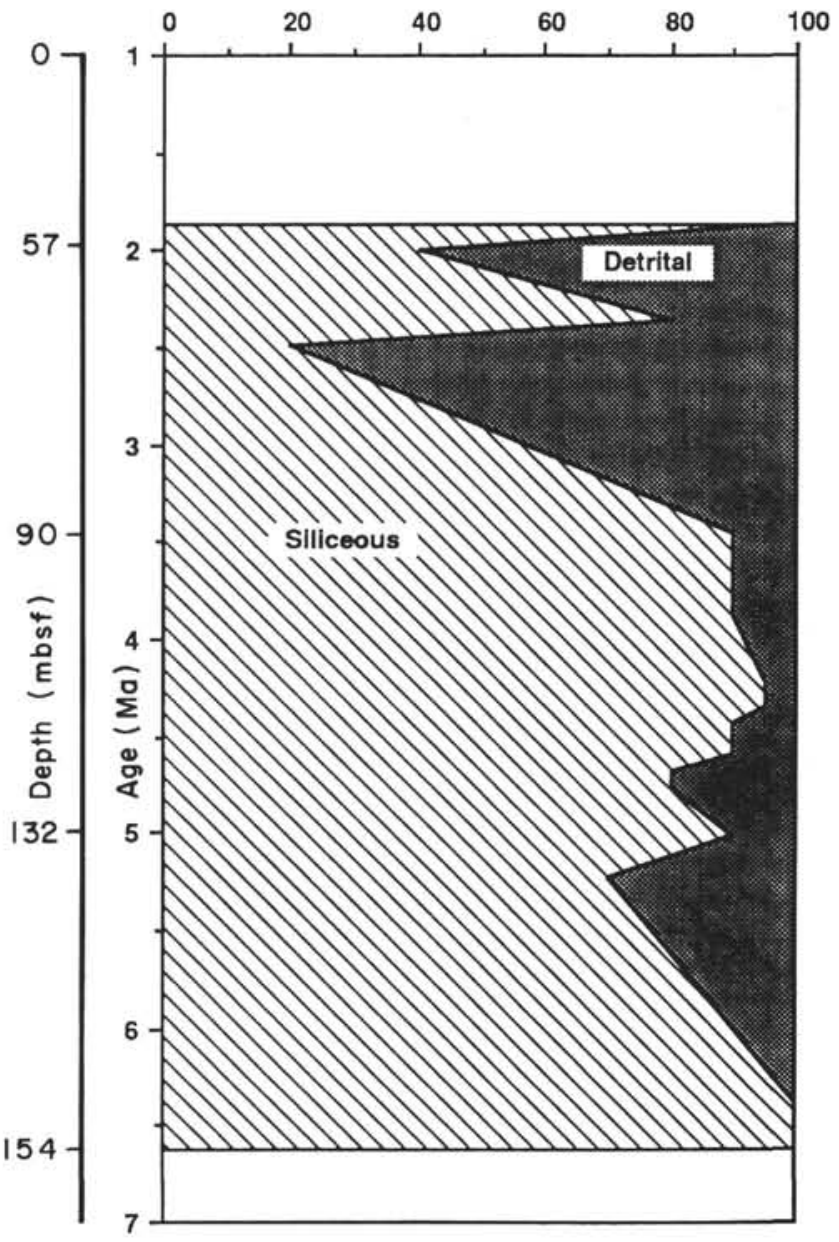

Figure 3. Principal components of the coarse $(>150 \mu \mathrm{m})$ fraction, Hole $701 \mathrm{C}$ (57-154 mbsf).

114-704A-2H-2, 114-116 cm). A sharp decrease in its abundance also occurs at about $2.5 \mathrm{Ma}$. In addition, we recognized two distinctive forms in the $G$. puncticulata population. The first one, $G$. aff. puncticulata, disappears at about $3.5 \mathrm{Ma}$ and could be considered as the ancestral form of $G$. puncticulata (see paleontological description). The frequency distribution of $G$. glutinata, which is present throughout the whole sequence, is about $5 \%$ to $10 \%$ prior to $2.5 \mathrm{Ma}$ and then decreases to less than $1 \%$ to the Holocene.

Two other species, Globigerina quinqueloba and Globorotalia scitula, are recognized at low percentages throughout the 
Components (\%)

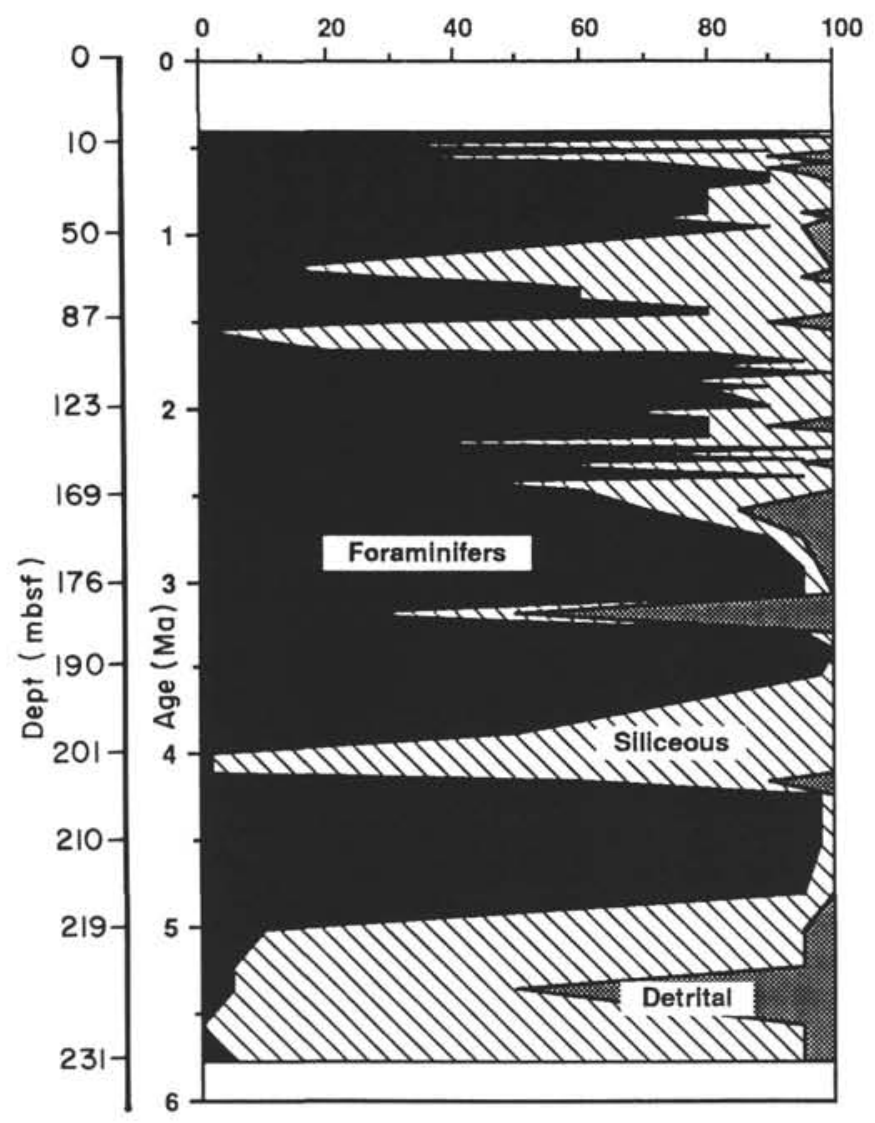

Figure 4. Principal components of the coarse $(>150 \mu \mathrm{m})$ fraction, Hole 704A (0-231 mbsf).

sequence (Fig. 6). Globorotalia inflata is present only in Core $114-704 \mathrm{~A}-1 \mathrm{H}$, at about $10 \%$ in the upper part. Rare Globorotalia crassaformis and Globorotalia truncatulinoides were also observed only in Core 114-704A-1H (Table 3).

\section{Hole 704B}

We examined only three samples from Core $114-704 \mathrm{~B}-4 \mathrm{H}$ (87.2\% recovery) and one from Core $114-704 \mathrm{~B}-5 \mathrm{H}(99.2 \%$ recovery), both of which were drilled with the APC (Appendix). The samples are dated to 0.8 to $0.6 \mathrm{Ma}$ (Ciesielski, Kristoffersen, et al., 1988) and correspond to lithostratigraphic Subunit IA, which is characterized by alternating calcareous siliceous and siliceous calcareous ooze. Planktonic foraminifers are the predominant constituent of the coarse fraction (Fig. 7). The most abundant species, $N$. pachyderma (left-coiling form), is associated with $G$. bulloides, G. quinqueloba, Globigerinita uvula, and G. puncticulata (Table 4). This type of Quaternary assemblage indicates subantarctic conditions similar to those at Hole 703A.

At about $0.7 \mathrm{Ma}$ (Sample 114-704B-4H-6, 40-42 cm), the quasimonospecific assemblage of $N$. pachyderma (left-coiling form) reflects cooler conditions. In this chronologically short sequence between 0.6 and $0.8 \mathrm{Ma} \mathrm{G}$. puncticulata is present, but contrary to Hole 114-704A-1H G. truncatulinoides and $G$. inflata are not represented.

\section{FORAMINIFERAL BIOSTRATIGRAPHY}

In the investigated sequences the low planktonic foraminiferal diversity results from geographic restriction to the subantarctic zone. Therefore, various planktonic markers species of the tropical or subtropical Neogene sequence (Berggren, 1977; Jenkins, 1975, 1978; Kennett and Srinivasan, 1983) cannot be used for biostratigraphy. In subantarctic deep-sea cores, Kennett (1970) and subsequently Keany and Kennett (1972) divided the subantarctic Pliocene-Quaternary sequence. These authors took into account the LAD of Globorotalia puncticulata and the first-appearance datum (FAD) of Globorotalia inflata and Globorotalia truncatulinoides. However, these datums are diachronous and depend on environmental adaptation in highlatitude biogeographic zones. For example, G. truncatulinoides appears in equatorial waters near the Olduvai Event (Berggren et al., 1987). But as a result of environmental adaptation it first appears later in northern waters (Weaver and Clement, 1987) and later still $(0.2 \mathrm{Ma})$ in subantarctic waters (Keany and Kennett, 1972). The same diachronous pattern also occurs for the other species, especially in areas close to the PFZ. The position of the sites below the CCD is a pernicious element for the study of planktonic foraminifers at Holes 701A and 701C, and Hole 704A appears to be the most useful for biostratigraphic discussion in spite of the lack of certain taxa and the paucity of others.

\section{Upper Miocene}

Miocene calcareous microfauna were detected only in Hole 704A. Globorotalia cf. sphericonomiozea occurs in Core $114-704 \mathrm{~A}-25 \mathrm{X}$. It is dated by the extrapolation of microfossil and paleomagnetic datums (Ciesielski, Kristoffersen, et al., 1988 ) to $5.3 \mathrm{Ma}$. This species is associated with Globorotalia cf. juanai.

\section{Pliocene}

$G$. cf. juanai disappears at the base of the lowermost Pliocene (Sample 114-704A-23X-5, 10-12 cm), at about 4.5 Ma. Globorotalia aff. puncticulata appears in abundance at about $5 \mathrm{Ma}$, and its LAD marks the top of the lowermost Pliocene (Sample 114-704A-21X-1, 70-72 cm) at about $3.4 \mathrm{Ma}$, close to the Gauss/Gilbert boundary (Ciesielski, Kristoffersen, et al., 1988). Globorotalia scitula, which is rare throughout the Pliocene sequence, is present in a peak of about $10 \%$ relative abundance.

The top of the Pliocene (Core 114-704A-11H) is not marked by any calcareous microfauna variation. The most important variation occurs at about $2.5 \mathrm{Ma}$. At this level, the frequency of $G$. puncticulata s. st. decreases as for Globigerina bulloides, whereas $N$. pachyderma (left-coiling form) increases in abundance. The frequency of Globigerina quinqueloba increases to more than $8 \%$ in Sample 114-704A-15H-6, 90-92 $\mathrm{cm}$, which is dated to about $2.16 \mathrm{Ma}$.

\section{Quaternary}

From Core 114-704A-11H (about $90 \mathrm{mbsf}$ ) to the upper section of the hole, the Quaternary planktonic foraminifer association changes little (Fig. 6). It is dominated by $N$. pachyderma (left-coiling form), with a frequency varying between $50 \%$ and more than $90 \%$. Diminution of the $N$. pachyderma percentage of the planktonic assemblage is linked mainly to an increase in abundance of the subantarctic species G. bulloides. This pattern is recognized in Samples 114-704A$3 \mathrm{H}-6,135-137 \mathrm{~cm}(0.6 \mathrm{Ma}), 114-704 \mathrm{~A}-1 \mathrm{H}-4,115-117 \mathrm{~cm}(0.44$ $\mathrm{Ma})$, and $114-704 \mathrm{~A}-1 \mathrm{H}-2,115-117 \mathrm{~cm}(0.4 \mathrm{Ma})$. 


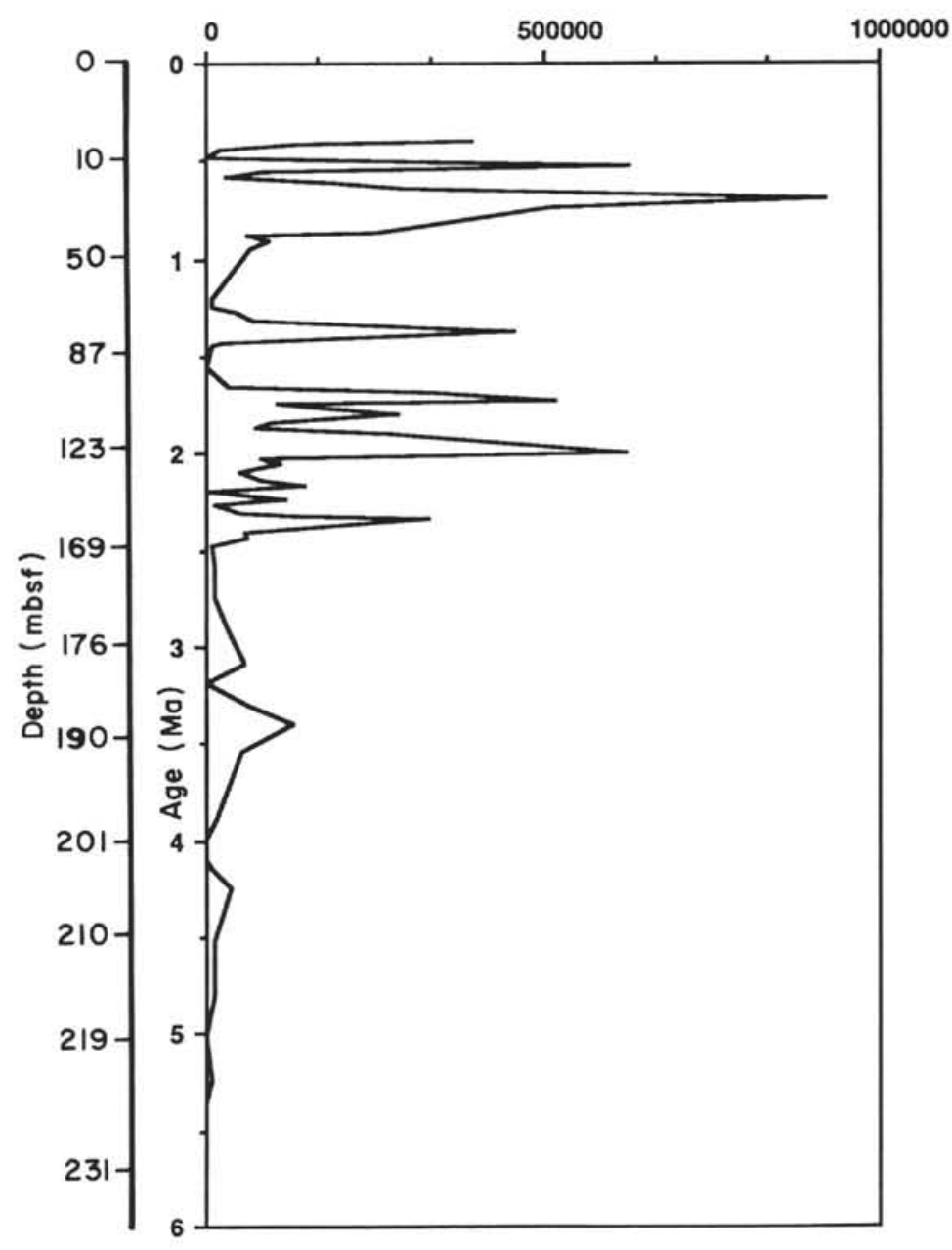

Figure 5. Abundance of planktonic foraminifers, Hole 704A.

G. puncticulata disappears in Sample 114-704A-2H-2, 114 $116 \mathrm{~cm}(0.48 \mathrm{Ma})$, just below the LAD of the nannoplankton species Pseudoemiliana lacunosa (Crux, this volume). $G$. quinqueloba increases in Sample 114-704A-4H-3, 10-12 cm, near the Brunhes/Matuyama boundary. The datum markers generally used, G. inflata, G. truncatulinoides, and Globorotalia crassaformis, have a brief occurrence in Core 114-704A$1 \mathrm{H}$ within the last $0.4 \mathrm{Ma}$. In Hole $703 \mathrm{~A}, \mathrm{G}$. truncatulinoides was observed only in Sample 114-703A-1H-2, 140-142 cm, at about 0.6 Ma.

\section{PALEOCEANOGRAPHY}

Large climatic variations are generally associated with variations in the volume of ice. In the South Atlantic, the buildup of major volumes of ice on the Antarctic continent is correlated with northward displacement of the polar planktonic microfauna (Kennett, 1970; Ciesielski, 1986). In the subantarctic area, therefore, climatic variations can be reflected by an increase of Neogloboquadrina pachyderma (left-coiling form). A high proportion of this variety $(90 \%-100 \%)$ indicates proximity of the PFZ, whereas south of the PFZ the foraminiferal fauna consists of $100 \% N$. pachyderma (left-coiling form) (Bé, 1977). Conversely, low proportions of this taxon and the increase of subpolar or transitional taxon, such as Globigerina bulloides, reflect a warming. The limited number of samples dealt with in this study is insufficient for drawing more detailed conclusions on the temporal variations in climate. The Pliocene-Quaternary sequences are characterized by alterations of cold-water faunas (left-coiling $N$. pachyderma) with cold- to intermediate surfacewater faunas (Fig. 6), which also include several other species ( $G$. bulloides and Globigerina quinqueloba). Within the Pliocene-Quaternary sequence, however, a noticeable cooling, as marked by the microfauna, occurs at about $2.5 \mathrm{Ma}$ (Fig. 8). This event could be linked with the development of Northern Hemisphere glaciation (Shackleton and Kennett, 1975). In the subantarctic South Atlantic this corresponds to a shift of the PFZ northward.

As $G$. quinqueloba is considered to be a surface-living species that is characteristic of nutrient-rich waters (Loubere, 1981), its increase at about 2.1 and $0.7 \mathrm{Ma}$ (Fig. 6) reflects an increase of upwelling and of biogenic productivity along the Antarctic Convergence.

\section{TAXONOMIC NOTES}

This nonsystematic list consists only of the species and forms recognized in the southern subantarctic sequences of the various Leg 114 holes investigated. For Quaternary species this order takes into account the present biogeographic distribution of the 

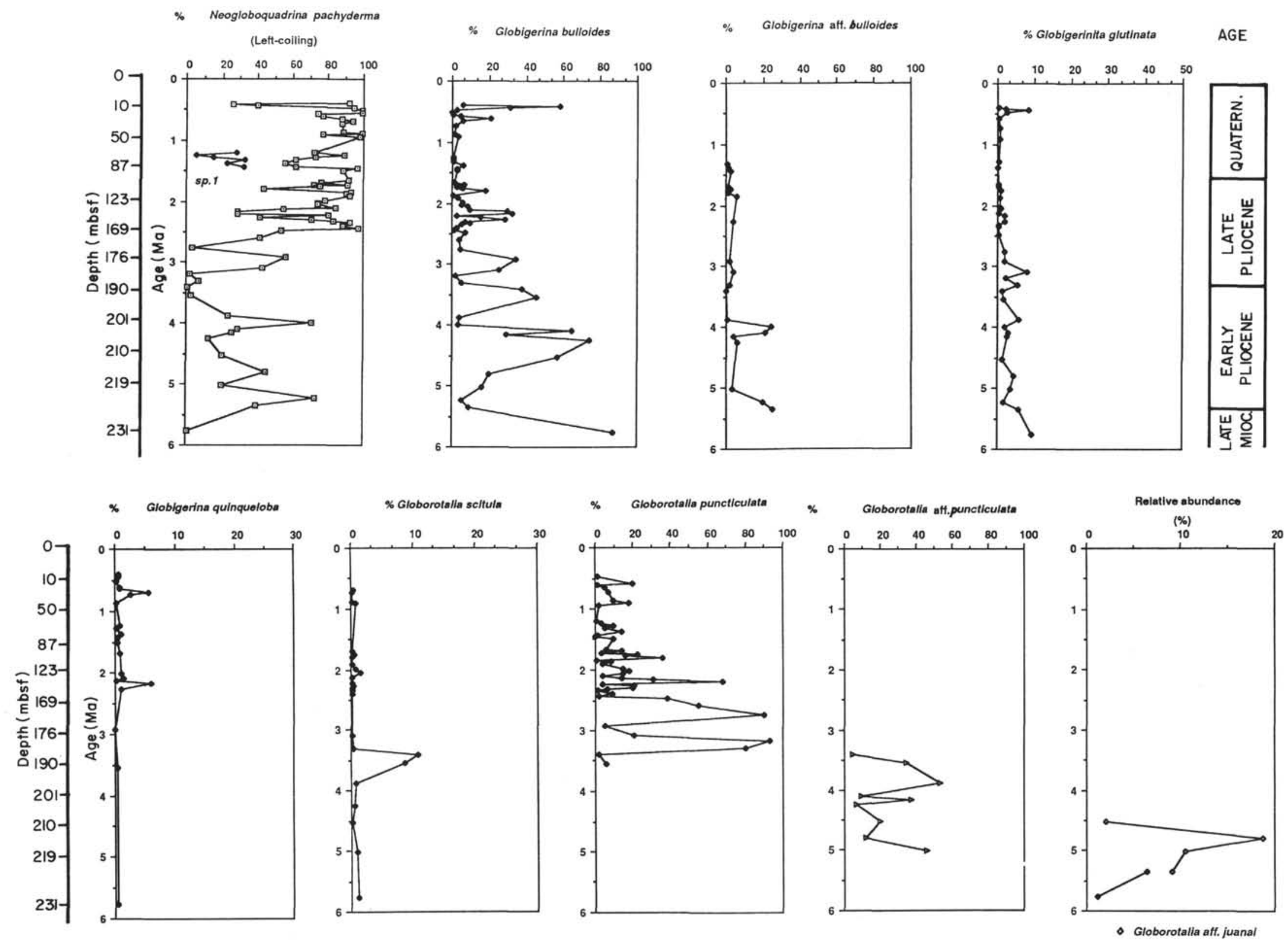

AGE

Figure 6. Distribution and relative abundance of some planktonic foraminifer species, Hole 704A. 


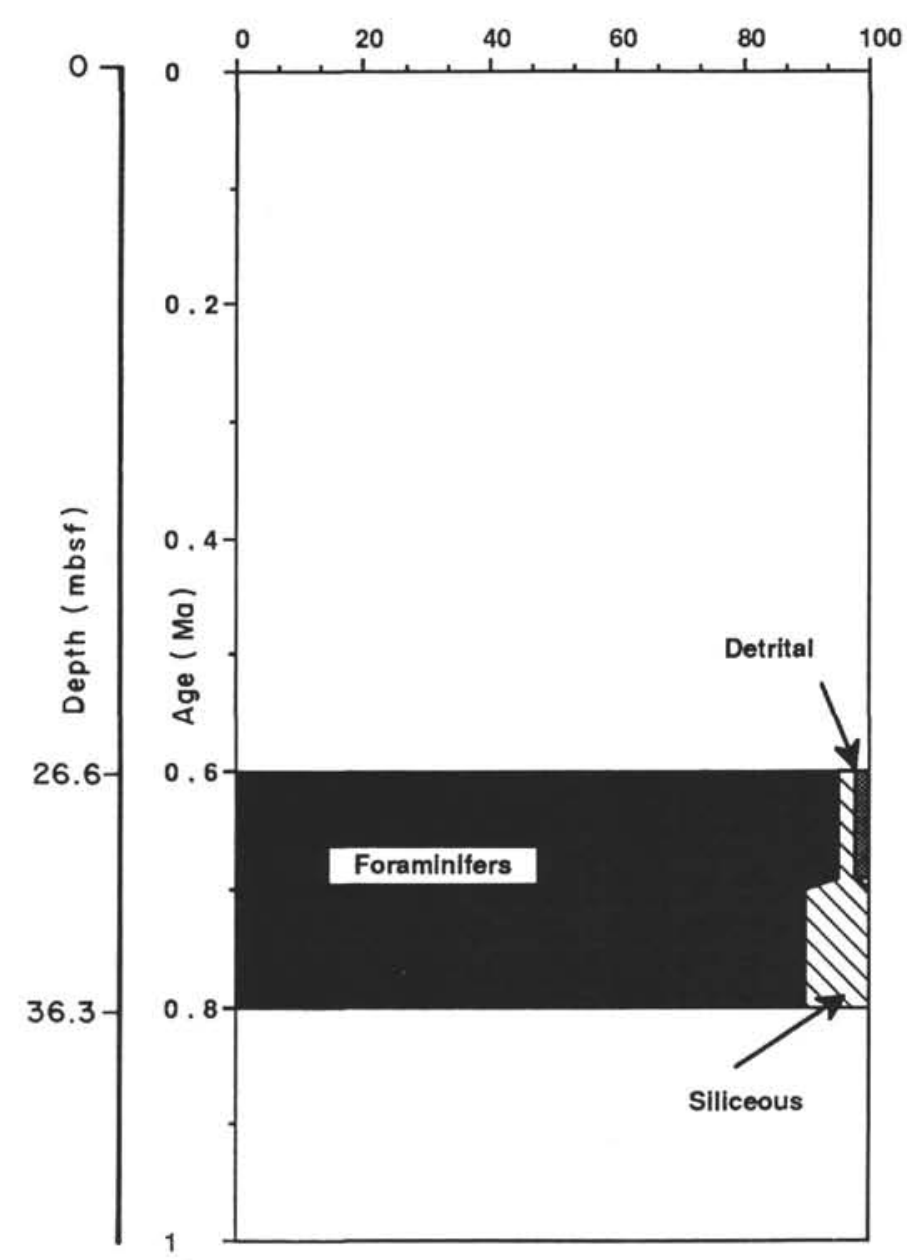

Figure 7. Principal components of the coarse $(>150 \mu \mathrm{m})$ fraction, Hole 704B.

species. The Neogene species are listed in alphabetical order. The figured specimens illustrated were deposited in the paleontological collection of the Department of Geology and Oceanography of the University of Bordeaux I.

\section{Quaternary Fauna}

Neogloboquadrina pachyderma (Ehrenberg) left coiling (PI. 2, Figs. 4-8) is the only species from the antarctic assemblage. Because it lives in cold water, the frequency of its occurrence is indicative of the temperature of the water. A monospecific assemblage is characteristic of deposition in antarctic water south of the PFZ.

In Cores $114-704 \mathrm{~A}-8 \mathrm{H}$ and $114-704 \mathrm{~A}-9 \mathrm{H}$ it was possible to differentiate in the left-coiling population a broader, more globose form, noted as $N$. pachyderma sp. 1.

Neogloboquadrina pachyderma (Ehrenberg) right coiling, although very rare in the upper Quaternary, is generally considered to be a subpolar form.

Globigerinita uvula (Ehrenberg) is very scarce in the coarse fraction.

Globigerina bulloides d'Orbigny (PI. 2, Fig. 3) is considered as an epipelagic species and inhabitant of temperate and cool waters. Different varieties could be differentiated from the type species in the population.
Globigerina aff. bulloides d'Orbigny (Pl. 1, Figs. 8 and 9) as described in Cores 114-704A-25X to 114-704A-9H is distinguished from $G$. bulloides $\mathrm{s}$. st. by a less globose but heavier test. The aperture is narrowed. The sutures between the chambers of the last whorl are deeply incised.

Globigerina quinqueloba (Natland) (Pl. 2, Fig. 9).

Globigerinita glutinata (Egger).

Globorotalia scitula (Brady).

Globorotalia inflata (d'Orbigny) (Pl. 2, Fig. 2) is common, but with a distribution restricted to the more recent sediments.

Globorotalia truncatulinoides (d'Orbigny) is rare, occurring only in the upper part of the Quaternary sequence.

Globorotalia crassaformis Galloway and Wissler (PI. 2, Fig. 1) is very rare.

Globorotalia puncticulata (Deshayes) (Pl. 1, Figs. 6 and 7) is assumed to have had a phylogenic transition with $G$. inflata in the late Pliocene (Scott, 1983; Wei and Kennett, 1988). The recovered sedimentary sequence does not give us the opportunity to document this evolution. These species do not live in the modern ocean.

Globorotalia aff. puncticulata (Deshayes) (PI. 1, Figs. 4 and 5) was recognized in the lowermost Pliocene of Hole $704 \mathrm{~A}$. This variety is differentiated from the type species by a more tightly coiled test. 


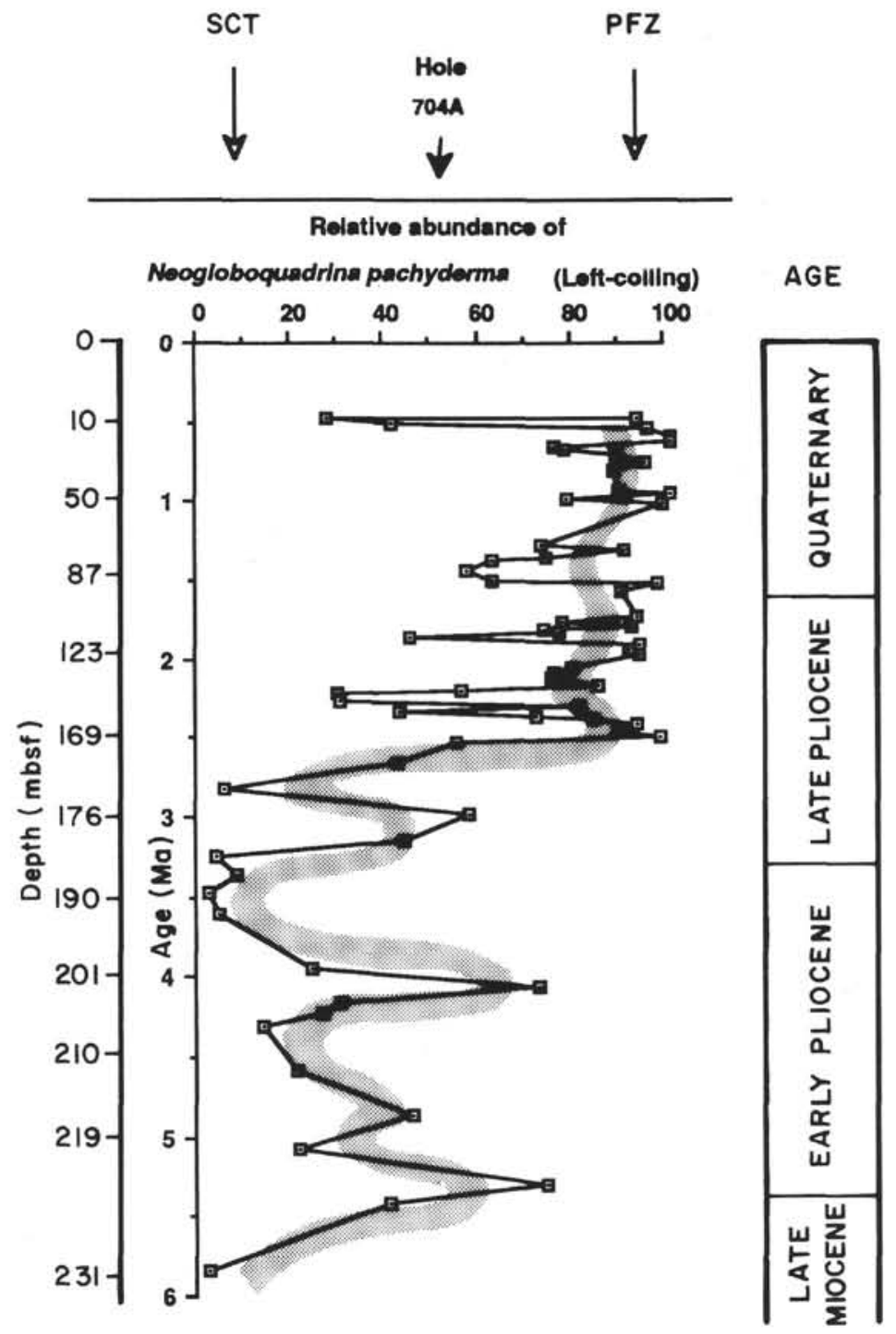

Figure 8. Relative abundance of Neogloboquadrina pachyderma (left-coiling form) in Hole 704A and the inferred positions of the PFZ and the Subtropical Convergence Zone (STC) with reference to the hole. The dotted curve corresponds to fluctuations in the PFZ.

The $G$. puncticulata population exhibits a pronounced sinistral coiling pattern.

\section{Neogene Species}

Globorotalia cf. juanai Bermudez (PI. 1, Fig. 3) is a tropical to temperate species.

Globorotalia cf. sphericonomiozea Walters (PI. 1, Figs. 1 and 2).

\section{CONCLUSIONS}

The analysis of planktonic foraminifers from upper Neogene sequences acquired during Leg 114 permitted us to recognize various faunal assemblages of about 16 species or varieties. Few species could be recognized from the upper Miocene sequence. The Pliocene sequence could be separated into an earlier part with the ancestral population of
Globorotalia puncticulata and a later part with $G$. puncticulata s. st.

The Pliocene-Quaternary foraminiferal record of Site 701 documents a climatic history characterized by oscillations of the PFZ over the site. During the late Quaternary, the PFZ was situated largely to the north of Site 701. The relatively continuous sedimentary record indicates quiet, basinal sedimentation throughout the observed section, although some minor perturbations are discernable in the foraminiferal assemblages. Sites 703 and 704 , on the Meteor Rise, contain a more complete and diversified history. The foraminiferal faunal patterns indicate significant cooling at $2.5 \mathrm{Ma}$, corresponding to a rapid shift of the PFZ toward the north (Fig. 8). Intervals of high biologic productivity are also evidenced at about 2.1 and $0.7 \mathrm{Ma}$ and indicate upwelling and a migration of the boundary of the Antarctic Convergence. 


\section{ACKNOWLEDGMENTS}

The writers wish to thank the ODP for making samples from Leg 114 available for the present study. We are indebted to Y. Kristoffersen for a number of pertinent corrections and suggestions as well as for an extremely careful polishing of the writing. Special thanks to the two anonymous reviewers, provided by ODP, for comments on a previous version of this manuscript. J. Chagnaud typed the manuscript and R. Anton did the original artwork. This investigation was supported by the Laboratoire de Géologie et d'Océanographie, Université de Bordeaux I.

\section{REFERENCES}

Bé, A.W.H., 1977. An ecological, zoogeographic and taxonomic review of Recent planktonic foraminifera. In Ramsay, A. (Ed.), Oceanic Micropaleontology (vol. 1): London (Academic Press), 1-100.

Berggren, W. A., 1977. Late Neogene planktonic foraminiferal biostratigraphy of the Rio Grande Rise (South Atlantic). Mar. Micropaleontol., 2:265-313.

Berggren, W. A., Kent, D. V., Flynn, J. J., and Van Couvering, J. A., 1987. Cenozoic geochronology. Geol. Soc. Am. Bull., 96:14071418.

Ciesielski, P. F., 1986. Pliocene variations in the position of the Antarctic Convergence in the Southwest Atlantic. Paleoceanography, 1:197-232.

Ciesielski, P. F., Kristoffersen, Y., et al., 1988. Proc. ODP, Init. Repts., 114: College Station, TX (Ocean Drilling Program).

Heusser, C. J., 1989. Polar perspective of late Quaternary climates in the Southern Hemisphere. Quat. Res. NY, 32:60-71.

Jenkins, D. G., 1975. Cenozoic planktonic foraminiferal biostratigraphy of the southwestern Pacific and Tasman Sea, DSDP Leg 29. In Kennett, J. P., Houtz, R. E., et al., Init. Repts. DSDP, 29: Washington (U.S. Govt. Printing Office), 449-467.
1978. Neogene planktonic foraminifers from DSDP Leg 40 Sites 360 and 362 in the southeastern Atlantic. In Bolli, H. M., Ryan, W.B.F., et al., Init. Repts. DSDP, 40: Washington (U.S. Govt. Printing Office), 723-739.

Keany, J., and Kennett, J. P., 1972. Pliocene-early Pleistocene paleoclimatic history recorded in antarctic-subantarctic deep sea cores. Deep-Sea Res., Part A, 19:529-548.

Kennett, J. P., 1970. Pleistocene paleoclimates and foraminiferal biostratigraphy in subantarctic deep-sea cores. Deep-Sea Res., Part A, 17:125-140.

Kennett, J. P., and Srinivasan, M. S., 1983. Neogene Planktonic Foraminifera: Stroudsburg, PA (Hutchinson Ross).

Loubere, P., 1981. Oceanographic parameters reflected in the sea bed distribution of planktonic foraminifera from the North Atlantic Mediterranean sea. J. Foraminiferal Res., 11:137-158

Scott, G. H., 1983. Gradual evolution in a planktonic foraminiferal lineage reconsidered. Paleobiology, 9:422-426.

Shackleton, N. J., and Kennett, J. P., 1975. Late Cenozoic oxygen and carbon isotopic changes at DSDP Site 284: implications for glacial history of the Northern Hemisphere and Antarctica. In Kennett, J. P., Houtz, R. E., et al., Init. Repts. DSDP, 29: Washington (U.S. Govt. Printing Office), 801-807.

Weaver, P.P.E., and Clement, B. M., 1987. Magnetobiostratigraphy of planktonic foraminiferal datums, Deep Sea Drilling Project Leg 94, North Atlantic. In Ruddiman, W. F., Kidd, R. B., Thomas, E., et al., Init. Repts. DSDP, 94: Washington (U.S. Govt. Printing Office), 815-829.

Wei, K.-Y., and Kennett, J. P., 1988. Phyletic gradualism and punctuated equilibrium in the late Neogene planktonic foraminiferal Globoconella. Paleobiology,, 14:345-363.

Date of initial receipt: 12 June 1989

Date of acceptance: 4 December 1989

Ms 114B-129 
APPENDIX

\begin{tabular}{|c|c|c|c|c|c|c|}
\hline $\begin{array}{l}\text { Core, section, } \\
\text { interval }(\mathrm{cm})\end{array}$ & $\begin{array}{l}\text { Depth } \\
\text { (mbsf) }\end{array}$ & $\begin{array}{l}\text { Age } \\
(\mathrm{Ma})\end{array}$ & $\begin{array}{l}\text { Fraction } \\
>150 \mu \mathrm{m} \\
(\%)\end{array}$ & $\begin{array}{l}\text { Foraminifers } \\
\qquad(\%)\end{array}$ & $\begin{array}{c}\text { Siliceous } \\
\text { component } \\
(\%)\end{array}$ & $\begin{array}{c}\text { Detrital } \\
\text { component } \\
(\%)\end{array}$ \\
\hline \multicolumn{7}{|l|}{ 114-701A- } \\
\hline $1 \mathrm{H}-3,28-30$ & 3.28 & 0.12 & 3 & 0 & 80 & 20 \\
\hline $1 \mathrm{H}-5,28-30$ & 6.78 & 0.21 & 3 & 0 & 100 & 0 \\
\hline $2 \mathrm{H}-1,30-32$ & 8.60 & 0.26 & 4 & 0 & 60 & 40 \\
\hline $2 \mathrm{H}-3,30-32$ & 11.60 & 0.36 & 2 & 0 & 80 & 20 \\
\hline $2 \mathrm{H}-5,30-32$ & 14.60 & 0.45 & 7 & 0 & 80 & 20 \\
\hline $3 \mathrm{H}-2,90-92$ & 20.20 & 0.88 & 1 & 0 & 95 & 5 \\
\hline $3 \mathrm{H}-4,90-92$ & 23.20 & 1.02 & 1 & 0 & 95 & 5 \\
\hline $3 \mathrm{H}-6,90-92$ & 26.20 & 1.15 & 10 & 5 & 50 & 45 \\
\hline $5 \mathrm{H}-4,34-36$ & 42.14 & 1.64 & 3 & 60 & 35 & 5 \\
\hline $5 \mathrm{H}-5,34-36$ & 43.64 & 1.7 & 4 & 20 & 50 & 30 \\
\hline $6 \mathrm{H}-3,38-40$ & 49.68 & 1.93 & 2 & 0 & 60 & 40 \\
\hline $6 \mathrm{H}-5,38-40$ & 52.68 & 2.03 & 3 & 0 & 50 & 50 \\
\hline $6 \mathrm{H}-7,38-40$ & 55.68 & 2.13 & 5 & 0 & 90 & 10 \\
\hline $7 \mathrm{H}-3,72-74$ & 59.52 & 2.29 & 3 & 0 & 80 & 20 \\
\hline $7 \mathrm{H}-4,120-122$ & 61.50 & 2.46 & 4 & 0 & 80 & 20 \\
\hline $8 \mathrm{H}-1,103-105$ & 66.33 & 2.66 & 2 & 0 & 60 & 40 \\
\hline $8 \mathrm{H}-3,103-105$ & 69.33 & 2.78 & 6 & 0 & 40 & 60 \\
\hline $8 \mathrm{H}-4,103-105$ & 70.83 & 2.84 & 2 & 0 & 90 & 10 \\
\hline \multicolumn{7}{|l|}{$114-701 C$} \\
\hline $7 \mathrm{H}-1,60-62$ & 54.5 & 1.86 & 3 & 0 & 95 & 5 \\
\hline $7 \mathrm{H}-3,60-62$ & 57.4 & 1.99 & 3 & 0 & 40 & 60 \\
\hline $8 \mathrm{H}-2,70-72$ & 65.5 & 2.35 & 5 & 0 & 80 & 20 \\
\hline $8 \mathrm{H}-4,70-72$ & 68.5 & 2.48 & 6 & 0 & 20 & 80 \\
\hline $10 \mathrm{H}-5,120-122$ & 89.5 & 3.45 & 1 & 0 & 90 & 10 \\
\hline $11 \mathrm{H}-3,115-117$ & 95.9 & 3.74 & 2 & 0 & 90 & 10 \\
\hline $11 \mathrm{H}-5,115-117$ & 98.9 & 3.87 & 2 & 0 & 90 & 10 \\
\hline $11 \mathrm{H}-7,10-12$ & 109.9 & 4.21 & 1 & 0 & 95 & 5 \\
\hline $13 \mathrm{H}-1,60-62$ & 111.4 & 4.25 & 2 & 0 & 95 & 5 \\
\hline $13 \mathrm{H}-3,60-62$ & 114.4 & 4.34 & 1 & 0 & 95 & 5 \\
\hline $13 \mathrm{H}-5,60-62$ & 117.4 & 4.43 & 2 & 0 & 90 & 10 \\
\hline $\begin{array}{l}14 \mathrm{H}-2 \\
104-106\end{array}$ & 122.8 & 4.59 & 2 & 0 & 90 & 10 \\
\hline $\begin{array}{l}14 \mathrm{H}-4 \\
104-106\end{array}$ & 125.8 & 4.68 & 1 & 0 & 80 & 20 \\
\hline $14 \mathrm{H}-6,104-106$ & 128.8 & 4.77 & 3 & 0 & 80 & 20 \\
\hline $15 \mathrm{H}-2,90-92$ & 132.2 & 5.01 & 1 & 0 & 90 & 10 \\
\hline $15 \mathrm{H}-4,90-92$ & 135.2 & 5.22 & 2 & 0 & 70 & 30 \\
\hline $17 \mathrm{H}-2,129-131$ & 151.6 & 6.38 & 4 & 0 & 100 & 0 \\
\hline $17 \mathrm{H}-4,129-131$ & 154.6 & 6.63 & 1 & 0 & 100 & 0 \\
\hline \multicolumn{7}{|l|}{$114-703 \mathrm{~A}-$} \\
\hline $1 \mathrm{H}-1,50-52$ & 0.5 & 0.1 & 40 & 95 & 0 & 5 \\
\hline $1 \mathrm{H}-2,140-142$ & 2.9 & 0.6 & 51 & 95 & 0 & 5 \\
\hline $1 \mathrm{H}-3,140-142$ & 4.4 & 0.8 & 47 & 95 & 0 & 5 \\
\hline \multicolumn{7}{|l|}{$114-704 \mathrm{~A}-$} \\
\hline $1 \mathrm{H}-1,114-116$ & 1.15 & 0.40 & 19 & 60 & 40 & 0 \\
\hline $1 \mathrm{H}-2,114-116$ & 2.65 & 0.41 & 26 & 70 & 30 & 0 \\
\hline $1 \mathrm{H}-4,135-137$ & 5.65 & 0.44 & 5 & 100 & 0 & 0 \\
\hline $2 \mathrm{H}-2,135-137$ & 9.80 & 0.48 & 1 & 10 & 90 & 0 \\
\hline $2 \mathrm{H}-4,135-137$ & 12.80 & 0.52 & 23 & 90 & 10 & 0 \\
\hline $2 \mathrm{H}-6,10-12$ & 15.80 & 0.55 & 9 & 10 & 80 & 10 \\
\hline $3 \mathrm{H}-2,10-12$ & 19.55 & 0.58 & 12 & 70 & 30 & 0 \\
\hline $3 \mathrm{H}-4,0-2$ & 22.55 & 0.61 & 19 & 80 & 10 & 10 \\
\hline $3 \mathrm{H}-6,0-2$ & 25.55 & 0.64 & 32 & 90 & 5 & 5 \\
\hline $4 \mathrm{H}-3,0-2$ & 29.30 & 0.69 & 27 & 90 & 10 & 0 \\
\hline $4 \mathrm{H}-6,0-2$ & 33.70 & 0.73 & 20 & 80 & 20 & 0 \\
\hline $5 \mathrm{H}-6,21-23$ & 43.20 & 0.86 & 18 & 80 & 20 & 0 \\
\hline $5 \mathrm{H}-7,21-23$ & 44.70 & 0.88 & 3 & 80 & 15 & 5 \\
\hline $6 \mathrm{H}-2,18-20$ & 46.70 & 0.91 & 16 & 70 & 30 & 0 \\
\hline $6 \mathrm{H}-4,76-78$ & 49.70 & 0.95 & 6 & 90 & 5 & 5 \\
\hline $8 \mathrm{H}-2,76-78$ & 65.91 & 1.20 & 2 & 10 & 90 & 0 \\
\hline $8 \mathrm{H}-4,30-32$ & 68.91 & 1.24 & 4 & 30 & 65 & 5 \\
\hline $8 \mathrm{H}-6,90-92$ & 71.88 & 1.28 & 8 & 50 & 50 & 0 \\
\hline $9 \mathrm{H}-1,90-92$ & 74.46 & 1.31 & 8 & 60 & 40 & 0 \\
\hline $9 \mathrm{H}-4,90-92$ & 78.96 & 1.37 & 13 & 60 & 40 & 0 \\
\hline $9 \mathrm{H}-7,104-106$ & 83.00 & 1.43 & 3 & 80 & 20 & 0 \\
\hline $10 \mathrm{H}-1,104-106$ & 84.10 & 1.45 & 2 & 80 & 20 & 0 \\
\hline $10 \mathrm{H}-3,47-49$ & 87.10 & 1.50 & 7 & 30 & 60 & 10 \\
\hline $10 \mathrm{H}-5,130-132$ & 90.10 & 1.55 & 1 & 0 & 100 & 0 \\
\hline $11 \mathrm{H}-1,130-132$ & 93.74 & 1.66 & 4 & 20 & 80 & 0 \\
\hline $11 \mathrm{H}-4,130-132$ & 97.24 & 1.69 & 11 & 80 & 20 & 0 \\
\hline $11 \mathrm{H}-7,90-92$ & 102.17 & 1.73 & 33 & 95 & 5 & 0 \\
\hline $12 \mathrm{H}-1,90-92$ & 103.50 & 1.74 & 12 & 80 & 20 & 0 \\
\hline
\end{tabular}


APPENDIX (continued).

\begin{tabular}{|c|c|c|c|c|c|c|}
\hline $\begin{array}{l}\text { Core, section, } \\
\text { interval }(\mathrm{cm})\end{array}$ & $\begin{array}{l}\text { Depth } \\
\text { (mbsf) }\end{array}$ & $\begin{array}{l}\text { Age } \\
(\mathrm{Ma})\end{array}$ & $\begin{array}{c}\text { Fraction } \\
>150 \mu \mathrm{m} \\
(\%)\end{array}$ & $\begin{array}{l}\text { Foraminifers } \\
\qquad(\%)\end{array}$ & $\begin{array}{c}\text { Siliceous } \\
\text { component } \\
\%\end{array}$ & $\begin{array}{c}\text { Detrital } \\
\text { component } \\
\%\end{array}$ \\
\hline $12 \mathrm{H}-3,90-92$ & 106.50 & 1.76 & 17 & 80 & 20 & 0 \\
\hline $12 \mathrm{H}-6,90-92$ & 109.50 & 1.80 & 15 & 100 & 0 & 0 \\
\hline $13 \mathrm{H}-1,90-92$ & 112.60 & 1.84 & 5 & 70 & 30 & 0 \\
\hline $13 \mathrm{H}-3,90-92$ & 115.60 & 1.87 & 8 & 90 & 10 & 0 \\
\hline $13 \mathrm{H}-5,90-92$ & 118.60 & 1.90 & 11 & 80 & 20 & 0 \\
\hline $14 \mathrm{H}-1,90-92$ & 122.10 & 1.99 & 16 & 90 & 10 & 0 \\
\hline $14 \mathrm{H}-3,90-92$ & 125.10 & 2.02 & 13 & 60 & 40 & 0 \\
\hline $14 \mathrm{H}-5,16-18$ & 128.10 & 2.05 & 46 & 80 & 20 & 0 \\
\hline $15 \mathrm{H}-2,16-18$ & 132.10 & 2.10 & 12 & 80 & 10 & 10 \\
\hline $15 \mathrm{H}-4,16-18$ & 135.10 & 2.13 & 12 & 80 & 20 & 0 \\
\hline $15 \mathrm{H}-6,116-118$ & 138.10 & 2.16 & 7 & 80 & 20 & 0 \\
\hline $16 \mathrm{H}-2,116-118$ & 140.16 & 2.20 & 5 & 5 & 95 & 0 \\
\hline $16 \mathrm{H}-4,116-118$ & 143.16 & 2.23 & 18 & 100 & 0 & 0 \\
\hline $16 \mathrm{H}-6,36-38$ & 146.16 & 2.26 & 4 & 60 & 40 & 0 \\
\hline $17 \mathrm{H}-1,36-38$ & 150.86 & 2.30 & 8 & 95 & 5 & 0 \\
\hline $17 \mathrm{H}-3,36-38$ & 153.86 & 2.34 & 19 & 60 & 40 & 0 \\
\hline $17 \mathrm{H}-5$ & 156.86 & 2.37 & 6 & 60 & 35 & 5 \\
\hline $18 X-2$ & 161.06 & 2.40 & 14 & 95 & 5 & 0 \\
\hline $18 X-4$ & 164.06 & 2.43 & 11 & 40 & 60 & 0 \\
\hline $18 X-6$ & 167.06 & 2.47 & 5 & 60 & 40 & 0 \\
\hline $19 X-1,130-132$ & 169.10 & 2.59 & 8 & 70 & 15 & 15 \\
\hline $19 X-3,130-132$ & 172.10 & 2.75 & 3 & 90 & 5 & 5 \\
\hline $19 X-5,70-72$ & 175.10 & 2.92 & 4 & 95 & 3 & 2 \\
\hline $20 \mathrm{X}-1,70-72$ & 179.50 & 3.08 & 9 & 95 & 5 & 0 \\
\hline $20 X-3,104-106$ & 182.50 & 3.18 & 1 & 20 & 30 & 50 \\
\hline $20 X-5,104-106$ & 185.50 & 3.30 & 8 & 95 & 5 & 0 \\
\hline $21 X-1,104-106$ & 187.40 & 3.40 & 19 & 100 & 0 & 0 \\
\hline $21 X-3,10-12$ & 190.40 & 3.54 & 16 & 98 & 2 & 0 \\
\hline $22 \mathrm{X}-1,10-12$ & 198.24 & 3.88 & 1 & 50 & 50 & 0 \\
\hline $22 \times-3,10-12$ & 201.24 & 3.99 & $i$ & 2 & 98 & 0 \\
\hline $22 X-5,0-2$ & 204.24 & 4.10 & 0 & 2 & 98 & 0 \\
\hline $23 \mathrm{X}-1,0-2$ & 206.80 & 4.15 & 2 & 60 & 30 & 10 \\
\hline $23 \mathrm{X}-3,0-2$ & 209.80 & 4.24 & 3 & 98 & 2 & 0 \\
\hline $23 X-5,0-2$ & 212.80 & 4.52 & 2 & 98 & 2 & 0 \\
\hline $24 X-1,0-2$ & 216.20 & 4.80 & 3 & 95 & 5 & 0 \\
\hline $24 X-3,0-2$ & 219.20 & 5.01 & 1 & 10 & 85 & 5 \\
\hline $24 X-5$ & 222.20 & 5.23 & 1 & 5 & 90 & 5 \\
\hline $25 \mathrm{X}-1$ & 225.70 & 5.35 & 1 & 5 & 45 & 50 \\
\hline $25 X-3$ & 228.70 & 5.56 & 0 & 0 & 95 & 5 \\
\hline $25 X-5$ & 231.70 & 5.77 & 0 & 5 & 90 & 5 \\
\hline \multicolumn{7}{|l|}{$114-704 \mathrm{~B}-$} \\
\hline $4 \mathrm{H}-2,40-42$ & 26.6 & 0.6 & 14 & 95 & 3 & 2 \\
\hline $4 \mathrm{H}-4,40-42$ & 29.6 & 0.69 & 33 & 95 & 3 & 2 \\
\hline $4 \mathrm{H}-6,40-42$ & 32.6 & 0.7 & 13 & 90 & 10 & 0 \\
\hline $5 \mathrm{H}-1,115-117$ & 36.3 & 0.8 & 23 & 90 & 10 & 0 \\
\hline
\end{tabular}




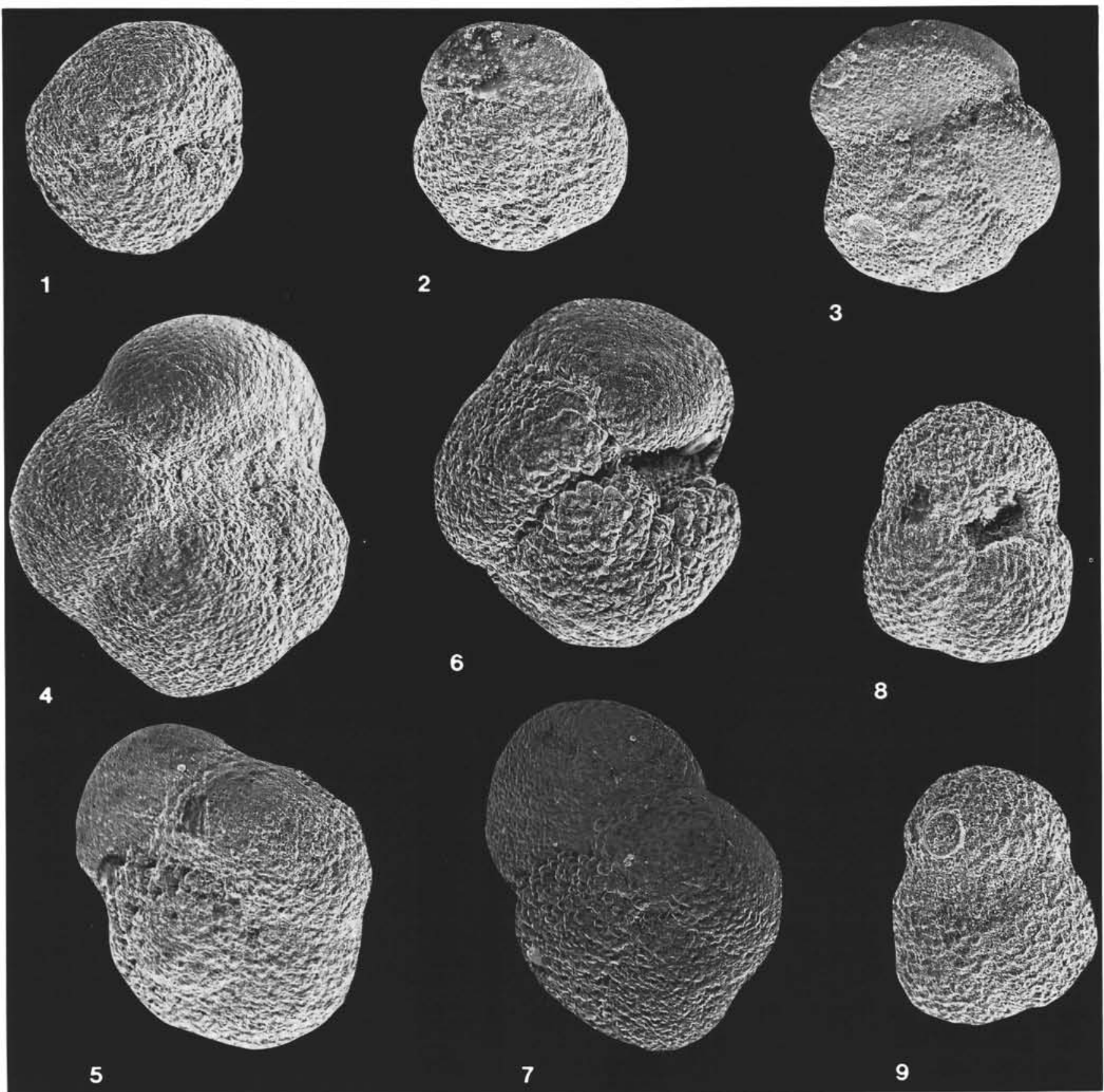

Plate 1. 1, 2. Globorotalia cf. sphericonomiozea Walters, Sample 114-704A-25X-1, 0-2 cm, $\times 130$, (1) umbilical view, (2) spiral view. 3. Globorotalia cf. juanai Bermudez, Sample 114-704A-25X-1, 0-2 cm, $\times 130$, spiral view. 4, 5. Globorotalia aff. puncticulata (Deshayes), Sample 114-704A-23X-5, 10-12 cm, $\times 130$, (4) umbilical view, (5) spiral view. 6, 7. Globorotalia puncticulata (Deshayes), Sample 114-704A-16H-6, 16-18 cm, $\times 105$, (6) umbilical view, (7) spiral view. 8, 9. Globigerina aff. bulloides d'Orbigny, Sample 114-704A-22X-5, 104-106 cm, $\times 130,(8)$ umbilical view, (9) distal view. 


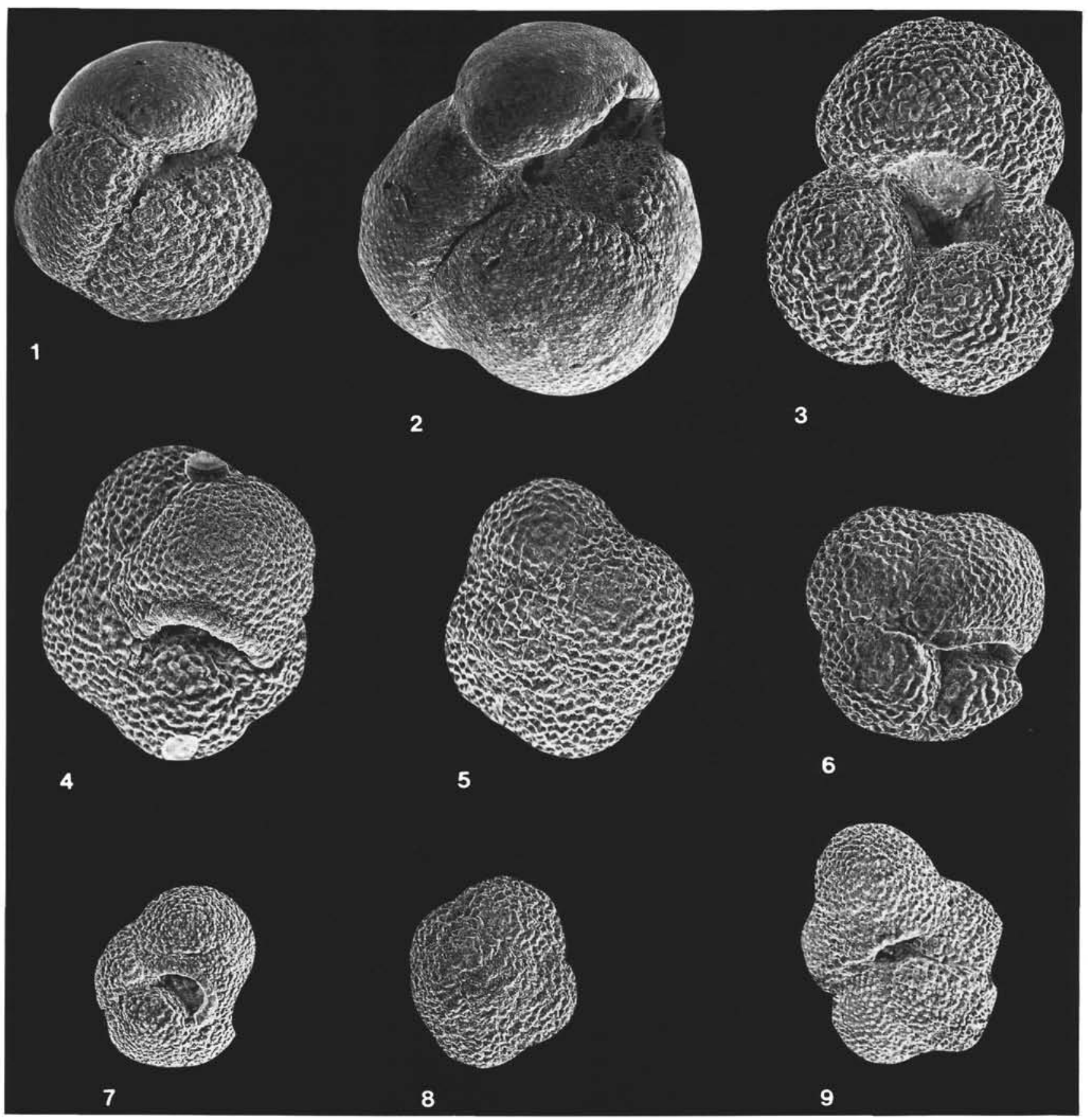

Plate 2. 1. Globorotalia crassaformis Galloway and Wissler, Sample $114-704 \mathrm{~A}-1 \mathrm{H}-4,115-117 \mathrm{~cm}, \times 100$, umbilical view. 2. Globorotalia inflata (d'Orbigny), Sample 114-704A-1H-4, 115-117 cm, $\times 100$, umbilical view. 3. Globigerina bulloides d'Orbigny, Sample 114-704A-1H-4, 115-117 cm, $\times 130$, umbilical view. 4-6. Neogloboquadrina pachyderma sp. 1 (Ehrenberg), left-coiling, Sample 114-704A-9H-7, 30-32 cm, $\times 130,(4,5)$ umbilical view, (6) distal view. 7, 8. Neogloboquadrina pachyderma (Ehrenberg) left-coiling, $\times 130$, Sample 114-704A-2H-2, 114-116 cm, (7) umbilical view, (8) distal view. 9. Globigerina quinqueloba Natland, Sample 114-704A-24-2, 114-116 cm, $\times 130$, umbilical view. 